2 Foundered lithospheric segments dropped into the mantle 3 transition zone beneath southern California

4 Youqiang $\mathrm{Yu}^{1}$, Stephen S. Gao ${ }^{2}$, Kelly H. Liu ${ }^{2}$, and Dapeng Zhao ${ }^{3}$

$5 \quad{ }^{1}$ State Key Laboratory of Marine Geology, Tongji University, Shanghai 200092, China. Email:

6 yuyouqiang@tongji.edu.cn

$7 \quad{ }^{2}$ Geology and Geophysics Program, Missouri University of Science and Technology, Rolla, MO

8 65409, USA

$9{ }^{3}$ Department of Geophysics, Graduate School of Science, Tohoku University, Sendai 980-8578, 10 Japan.

\title{
11 Introduction
}

12 This document contains detailed descriptions of the data and methods used to obtain the 13 results presented in the paper, as well as Supplementary Figures DR1-8 and Table DR1. This

14 material is associated with Yu et al., Foundered lithospheric segments dropped into the mantle

15 transition zone beneath southern California.

\section{Data and Receiver Function}

All the available three-component broad-band seismic data used in this study were requested

18 from the Incorporated Research Institutions for Seismology (IRIS) Data Management Center

19 (DMC) and recorded at a total of 476 stations over the period of 1988-2017 (Figure 1A). The

20 stations are evenly distributed except for three dense linear profiles transecting the Great Valley,

21 the Transverse Ranges, the Peninsula Ranges and the Salton Trough. Teleseismic events with

22 epicentral distances falling in a range of $30^{\circ}-100^{\circ}$ were selected based on a cut-off magnitude

23 of $M c$ defined as $M c=5.2+(D e-30) /(180-30)-H e / 700$, where $D e$ and He indicate the

24 epicentral distance in degree and the focal depth in km, respectively (Liu and Gao, 2010). A

25 four-pole two-pass Bessel filter in the frequency band of 0.02-0.2 Hz was utilized to improve

26 signal-to-noise ratios (SNRs) of the original seismograms. We applied a set of exponential

27 weighting functions with a window width of $60 \mathrm{~s}$ and centered on the theoretical PP arrivals

28 predicted from the IASP91 Earth model to diminish its degenerating effects on the resulting RFs

29 (Gao and Liu, 2014a). The filtered seismograms with an SNR of the first arrival exceeding 4 on 
the vertical component were converted into radial RFs using the frequency-domain water-level

31 deconvolution procedure (Ammon, 1991) with a water-level value of 0.03. An SNR-based

32 procedure was employed to reject those RFs with high noise or abnormal arrivals in the P-wave

33 coda (sea Gao and Liu, 2014b for more details of the procedure). As a result, we obtained a total

34 of 86,261 high-quality RFs from 3897 teleseismic events (Figure 1).

35 The geographic coordinates of the ray-piercing points for each of the RFs were computed at

36 approximately the middle of the MTZ $(535 \mathrm{~km})$ based on the 1-D IASP91 Earth model. The

37 study area was evenly divided into overlapping bins with a circular radius of $0.3^{\circ}$. Similar grid

38 intervals of $0.25^{\circ}$ (Cao et al., 2010) and $0.5^{\circ}$ (Tauzin et al., 2013) were employed to study the

39 western US and that of $0.4^{\circ}$ for southern California (Simmons and Gurrola, 2000). We then

40 employ a more accurate technique developed by Gao and Liu (2014a) to conduct moveout-

41 correction and stacking of all the RFs in a bin with a depth range of 350-800 km and a vertical

42 resolution of $1 \mathrm{~km}$ using the 1-D IASP91 Earth model. Such a procedure is under the assumption

43 of the non-plane wave front and takes the difference in ray parameters between the direct P-wave

44 and the converted S-wave into consideration. Bins with less than $100 \mathrm{RFs}$ were rejected and the

45 number of RFs within each bin ranges from 100 to 8378. The mean and standard deviation of the

46 discontinuity depths is quantified by adopting a bootstrap resampling approach with 50

47 resampling iterations (Efron and Tibshirani 1986) for RFs within each of the bins. The resulting

48 depth series were manually checked, and prominent peaks are clearly visible around the depths

49 of d410 and d660 (Figure DR1). Following the same procedures above, RFs with their piercing

50 points falling in the four subareas characterized as significant MTZ thickening were individually

51 migrated and stacked to further confirm the resulting MTZ features (Figure DR3). Results from

52 RF stacking with a larger circular radius of $0.5^{\circ}$ are also offered (Figure DR4).

53 Estimation of Average Vp Anomaly above the d410 Depth

$54 \quad$ P-wave velocity $(\mathrm{Vp})$ anomalies derived from teleseismic tomography are relative to the

55 mean velocity at each depth under the study region due to the employment of relative instead of

56 absolute travel-time residuals (e.g., Zhao et al., 2006; Foulger et al., 2013). Thus, it becomes

57 difficult to utilize the obtained 3-D velocity model to determine the average absolute- $\mathrm{Vp}$

58 anomaly above the $\mathrm{d} 410 \mathrm{depth}$, which is a significant indicator on the generally tectonic setting

59 of the study area. The apparent $\mathrm{d} 410$ depths $\left(D_{A}\right)$ are mostly attributed to the cumulative effects

60 of velocity anomalies in the crust and upper mantle, and can be employed to determine the 
average $\mathrm{Vp}$ anomaly by analyzing their net differences relative to the assumed true depth of 410

$62 \mathrm{~km}\left(D_{T}\right)$. Given a particular Vp anomaly $\left(\delta V_{P}\right), \delta V_{S}$ can be obtained by assuming a $\gamma$ factor

63 based on equation (Gao and Liu, 2014a)

$$
\frac{\delta V_{S}}{V_{S 0}}=\gamma \frac{\delta V_{P}}{V_{P 0}},
$$

where $V_{P 0}$ and $V_{S 0}$ are the mean $\mathrm{P}$ and $\mathrm{S}$ wave velocities, respectively, above the $\mathrm{d} 410 \mathrm{depth}$ according to the 1-D IASP91 Earth model; $\delta V_{P}$ and $\delta V_{S}$ are the absolute $\mathrm{P}$ and $\mathrm{S}$ wave velocity anomalies, respectively. The $\gamma$ value is assigned as 2.15 , which was estimated for the western United States (Gao and Liu, 2014b).

The apparent d410 depth $\left(D_{A}^{\prime}\right)$ can be theoretically obtained by (Gao and Liu, 2014a)

$$
D_{A}^{\prime}=\frac{V_{P 0}+\delta V_{P}-V_{S 0}-\delta V_{S}}{\left(V_{S 0}+\delta V_{S}\right) \times\left(V_{P 0}+\delta V_{P}\right)} \times \frac{V_{P 0} \times V_{S 0}}{V_{P 0}-V_{S 0}} D_{T},
$$

The calculated $D_{A}^{\prime}$ for a given $\delta V_{P}$ is input to determine the standard deviation $\left(\sigma\left(\delta V_{P}\right)\right)$ relative to the observed $\mathrm{d} 410$ depths using

$$
\sigma\left(\delta V_{P}\right)=\sqrt{\frac{1}{n} \sum_{i=1}^{n}\left[D_{A}^{\prime}\left(\delta V_{P}\right)-D_{A}(i)\right]^{2}},
$$

74 where $n$ is the number of bins and $D_{A}(i)$ corresponds to the observed d410 depth in the $i$ th bin. A 75 series of $\mathrm{Vp}$ anomalies ranging from $-3 \%$ to $0 \%$ are incorporated to determine $\sigma\left(\delta V_{P}\right)$ using

76 Equation (3) with the minimum value of $\sigma\left(\delta V_{P}\right)$ corresponding to the optimal $\delta V_{P}$, which

77 indicates the mean $\mathrm{Vp}$ anomaly above the $\mathrm{d} 410$ including the crust and upper mantle beneath the 78 study region (Figure 2D).

\section{References for Data Repository}

80 Ammon, C.J., 1991, The isolation of receiver effects from teleseismic Pwaveforms: Bulletin of 81 the Seismological Society of America, v. 81, p. 2504-2510.

82 Burdick, S., Vernon, F.L., Martynov, V., Eakins, J., Cox, T., Tytell, J., Mulder, T., White, M.C., 83 Astiz, L., Pavlis, G.L., and van der Hilst, R.D., 2017, Model update May 2016: Upper84 mantle heterogeneity beneath North America from travel-time tomography with global and 85 USArray data: Seismological Research Letters, v. 88(2A), p.319-325. 
Cao, A., and Levander, A., 2010, High-resolution transition zone structures of the Gorda Slab beneath the western United States: Implication for deep water subduction: Journal of Geophysical Research, v. 115(B7), p. B07301, https://doi.org/10.1029/2009JB006876.

Foulger, G.R., Panza, G.F., Artemieva, I.M., Bastow, I.D., Cammarano, F., Evans, J.R.,

Gao, S.S., and Liu, K.H., 2014a, Imaging mantle discontinuities using multiply-reflected P-to-S

Gao, S.S., and Liu, K.H., 2014b, Mantle transition zone discontinuities beneath the contiguous

Schmandt, B., and Humphreys, E., 2010, Seismic heterogeneity and small-scale convection in

Efron, B., and Tibshirani, R., 1986, Bootstrap methods for standard errors, confidence intervals, and other measures of statistical accuracy: Statistical Science, v. 1, p. 54-75. Hamilton, W.B., Julian, B.R., Lustrino, M., Thybo, H., and Yanovskaya, T.B., 2013, Caveats on tomographic images: Terra Nova, v. 25(4), p.259-281. conversions: Earth and Planetary Science Letters, v. 402, p. 99-106, http://doi .org /10 .1016 /j .eps1 .2013.08.025. United States: Journal of Geophysical Research, v. 119, p. 6452-6468, http://doi .org /10 .1002 /2014JB011253.

Liu, K.H., and Gao, S.S., 2010, Spatial variations of crustal characteristics beneath the Hoggar swell, Algeria revealed by systematic analyses of receiver functions from a single seismic station: Geochemistry Geophysics Geosystems, v. 11, p. Q08011, http://doi .org /10.1029/2010GC003091.

the southern California upper mantle: Geochemistry Geophysics Geosystems, v. 11, p. Q05004, https://doi.org/10.1029/2010GC003042.

Schmandt, B., and Lin, F.C., 2014, P and S wave tomography of the mantle beneath the United States: Geophysical Research Letters, v. 41(18), p.6342-6349, https://doi.org/10.1002/2014GL061231.

Simmons, N.A., and Gurrola, H., 2000, Multiple seismic discontinuities near the base of the transition zone in the Earth's mantle: Nature, v. 405, p. 559-562.

Tauzin, B., Van Der Hilst, R.D., Wittlinger, G., and Ricard, Y., 2013, Multiple transition zone seismic discontinuities and low velocity layers below western United States: Journal of Geophysical Research, v. 118(5), p. 2307-2322, https://doi.org/10.1002/jgrb.50182. 
115 Zhao, D., Lei, J., Inoue, T., Yamada, A., and Gao, S.S., 2006, Deep structure and origin of the 116 Baikal rift zone: Earth and Planetary Science Letters, v. 243, p. 681-691, 117 https://doi.org/10.1016/j.eps1.2006.01.033. 

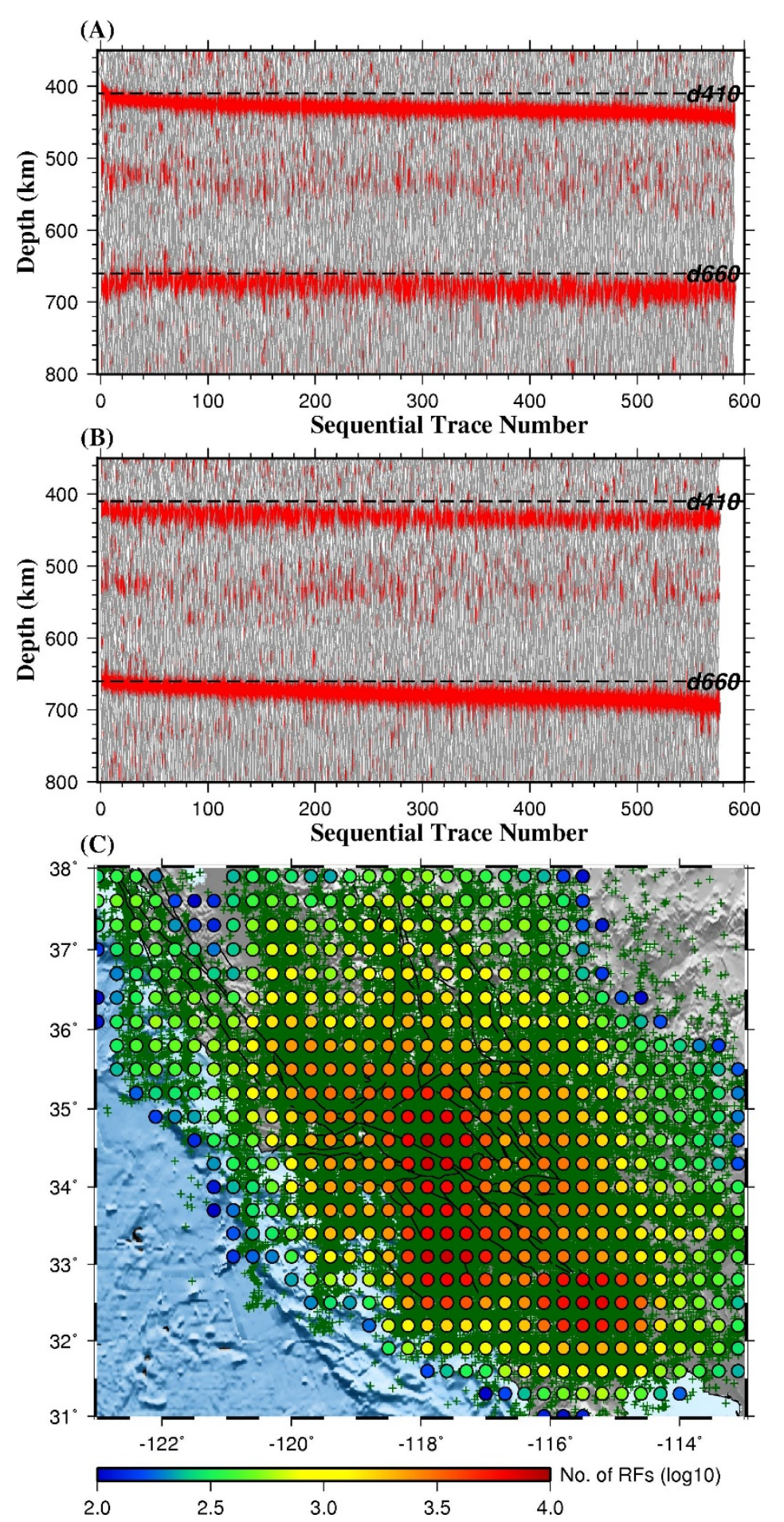

120 Figure DR1. Binned RF traces sorted based on the sequentially increasing apparent depth of the

121 valid d410 (A) and d660 (B), respectively. (C): Number of RFs in each bin. The color of the

122 circles indicates the quantity of RFs per bin. The green plus symbols denote ray piercing points

123 at $535 \mathrm{~km}$ depth. 


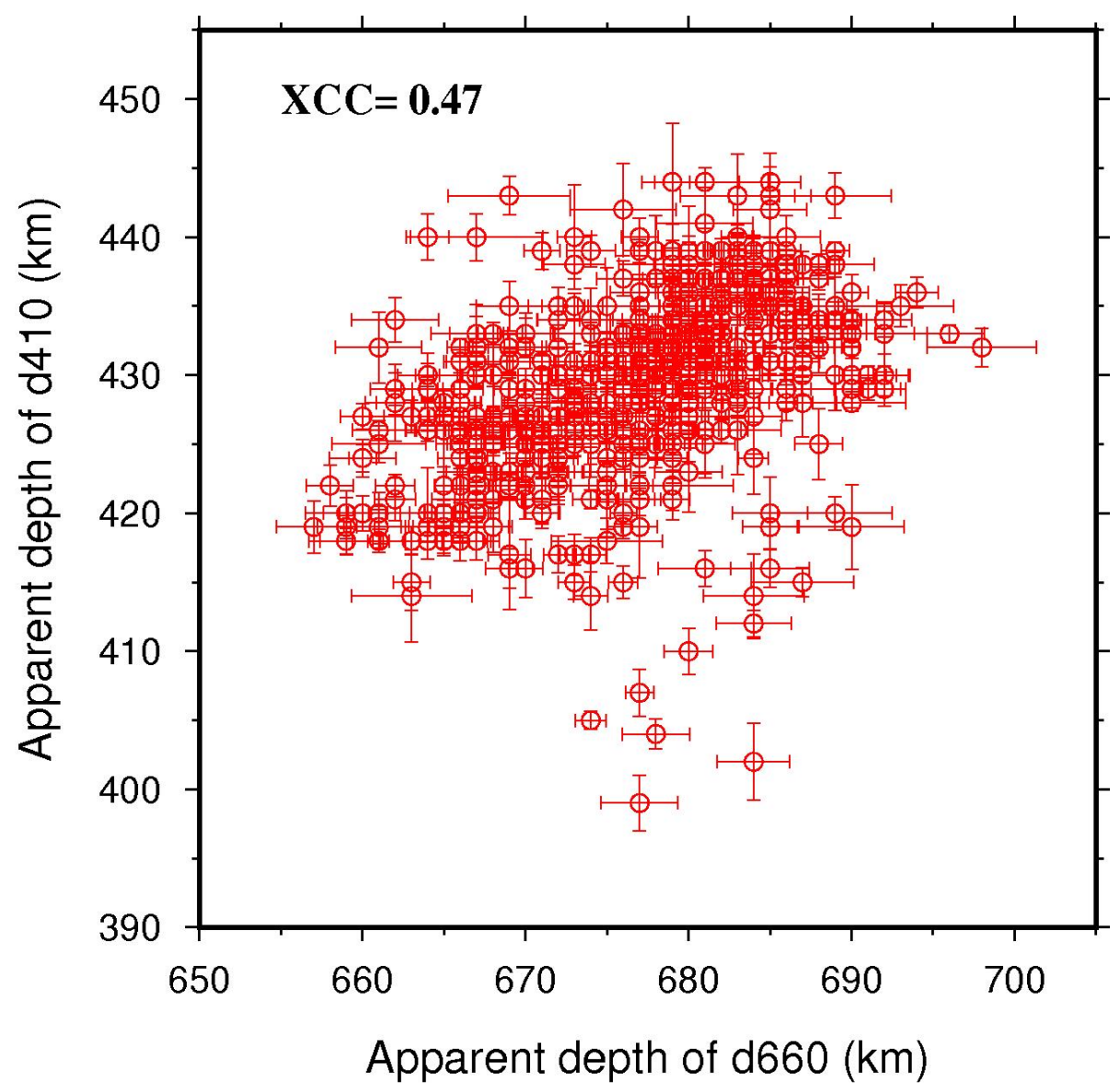

125 Figure DR2. Apparent d410 depths plotted against apparent d660 depths with a cross-correlation

126 coefficient (XCC) of 0.47 . 
(A)

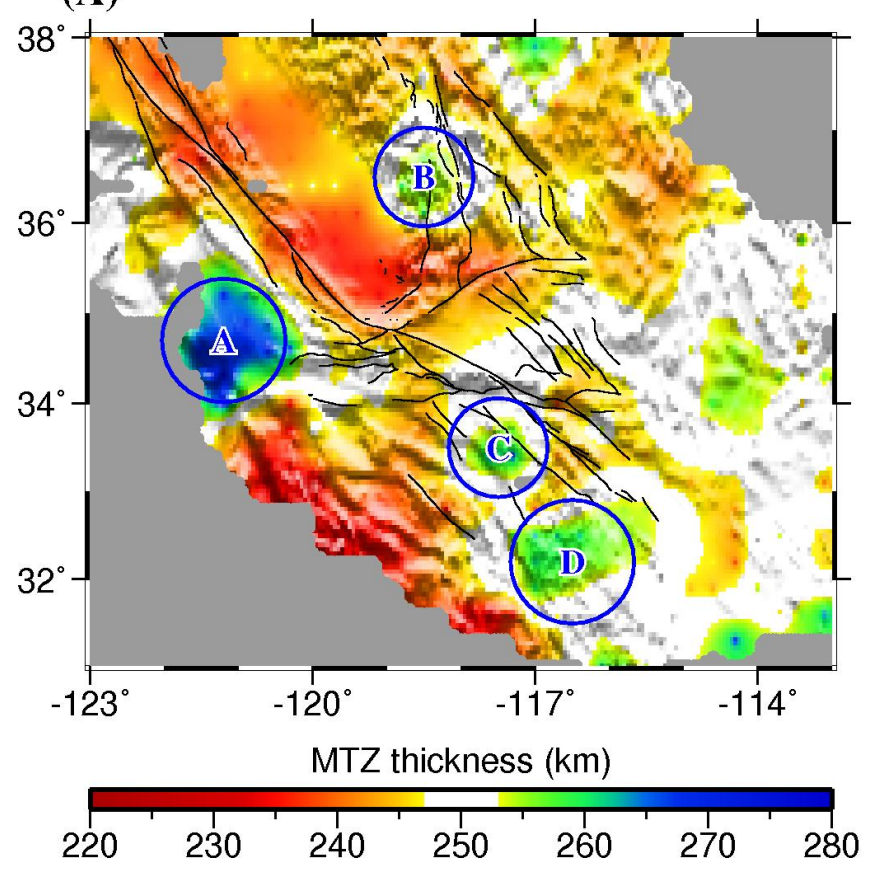

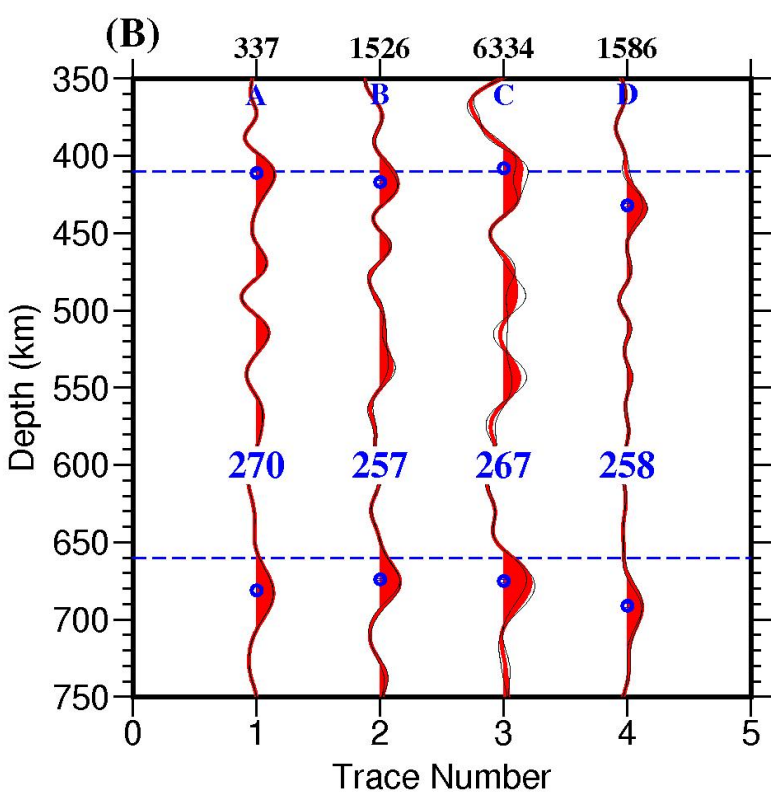

Figure DR3. Results of RF stacking at four subareas. A: Spatial distribution of the resulting 129 mantle transition zone (MTZ) thickness measurements. B: Resulting depth series by stacking

130 RFs within each subareas in A indicated by the blue letters at the top. The black numbers at the

131 top and the blue ones in the middle indicate the number of RFs and the mean MTZ thickness, 132 respectively. 
(A)

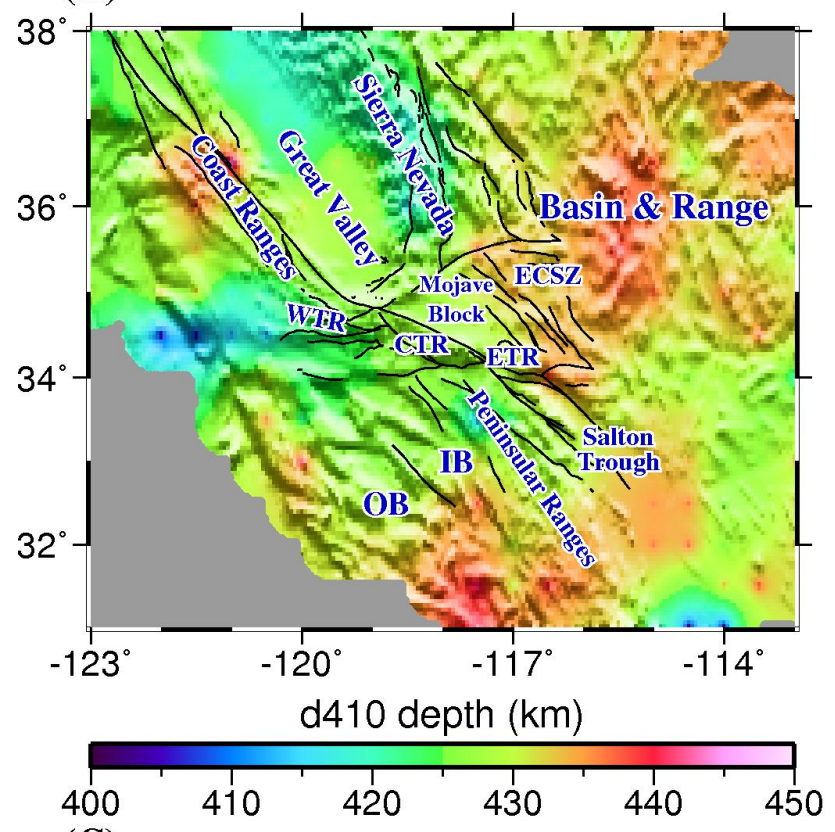

(C)
(B)

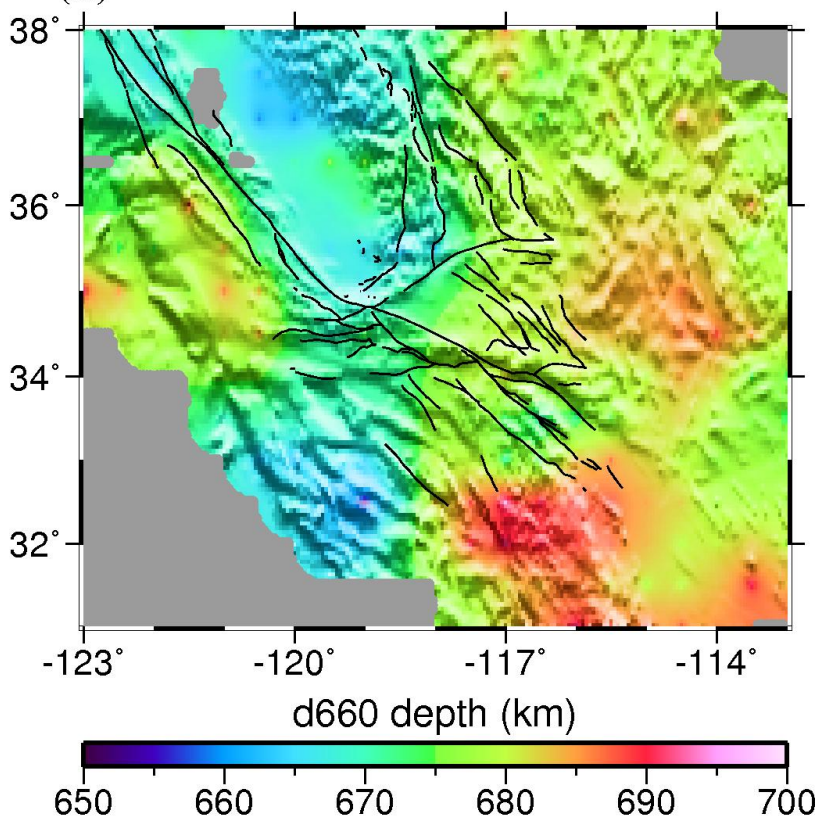

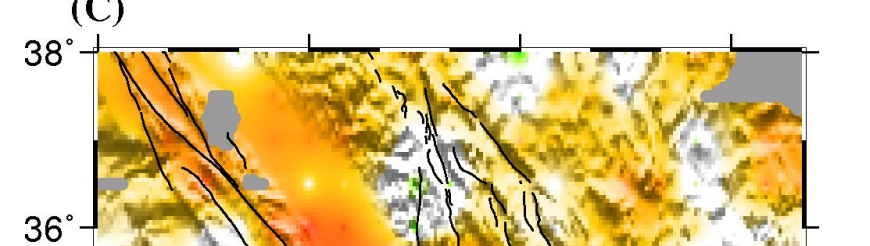
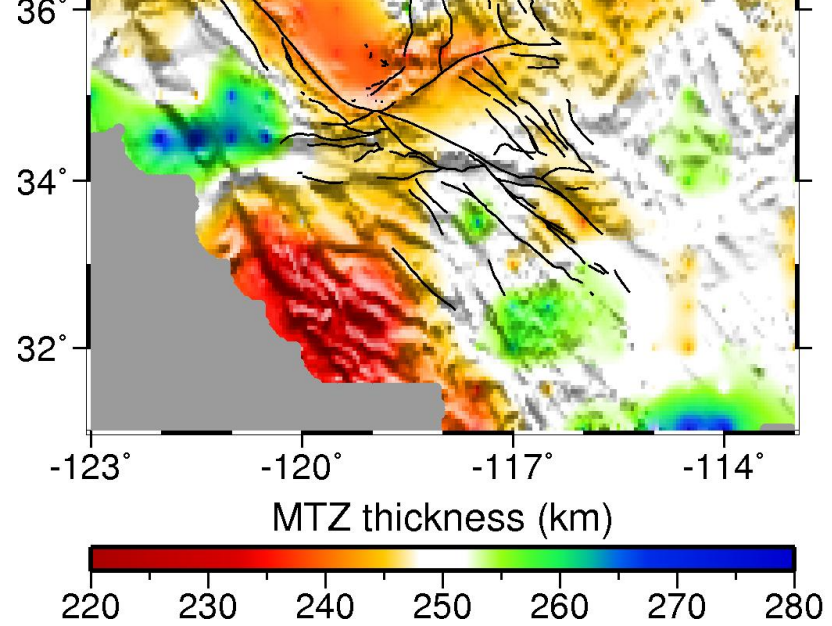

134 Figure DR4. Same as Figure 2 but for resutls with RF stacking interval of 0.5 degrees. 


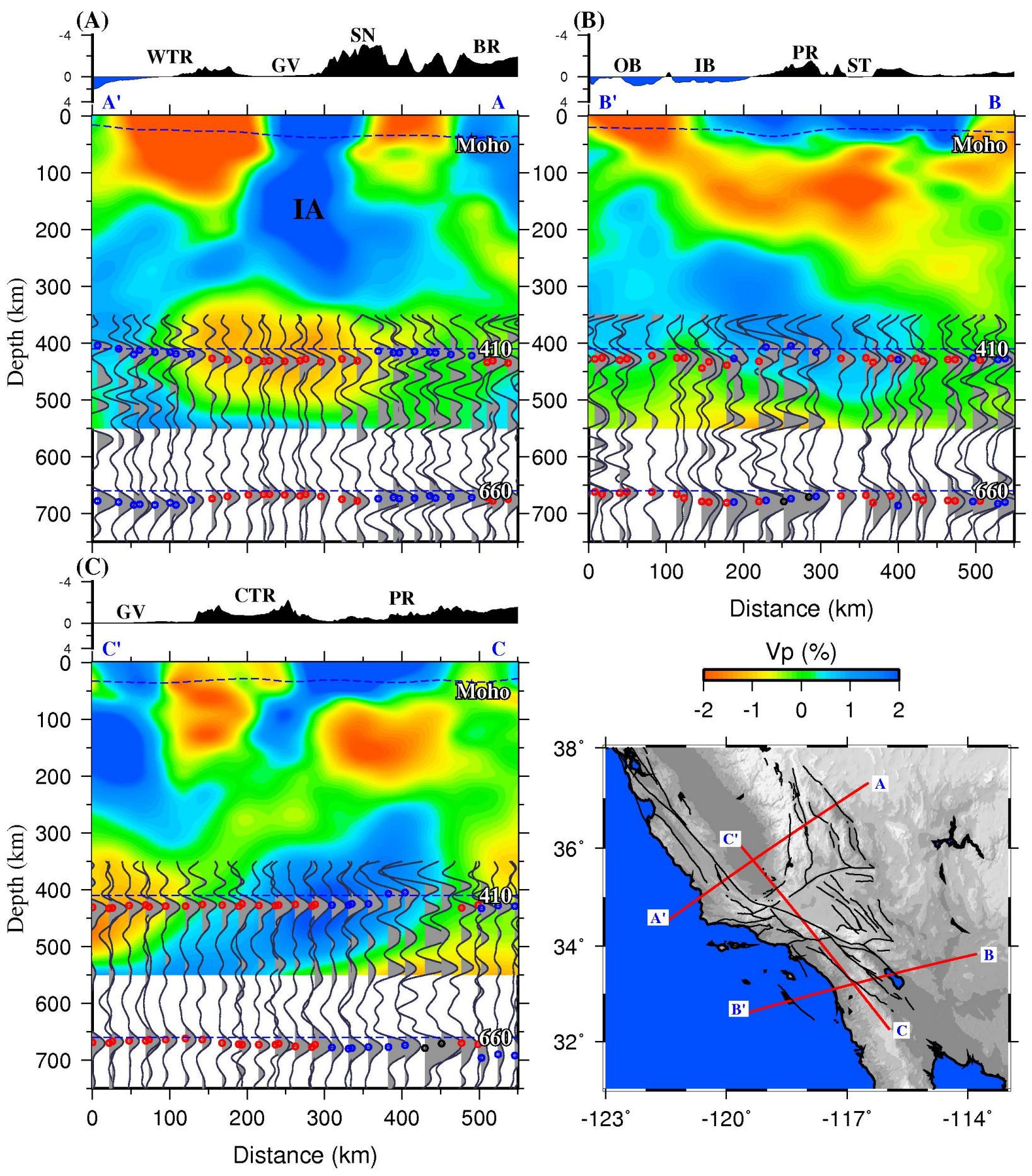

136 Figure DR5. Same as Figure 3 but for P-wave velocity model of Schmandt and Humphreys 137 (2010). 


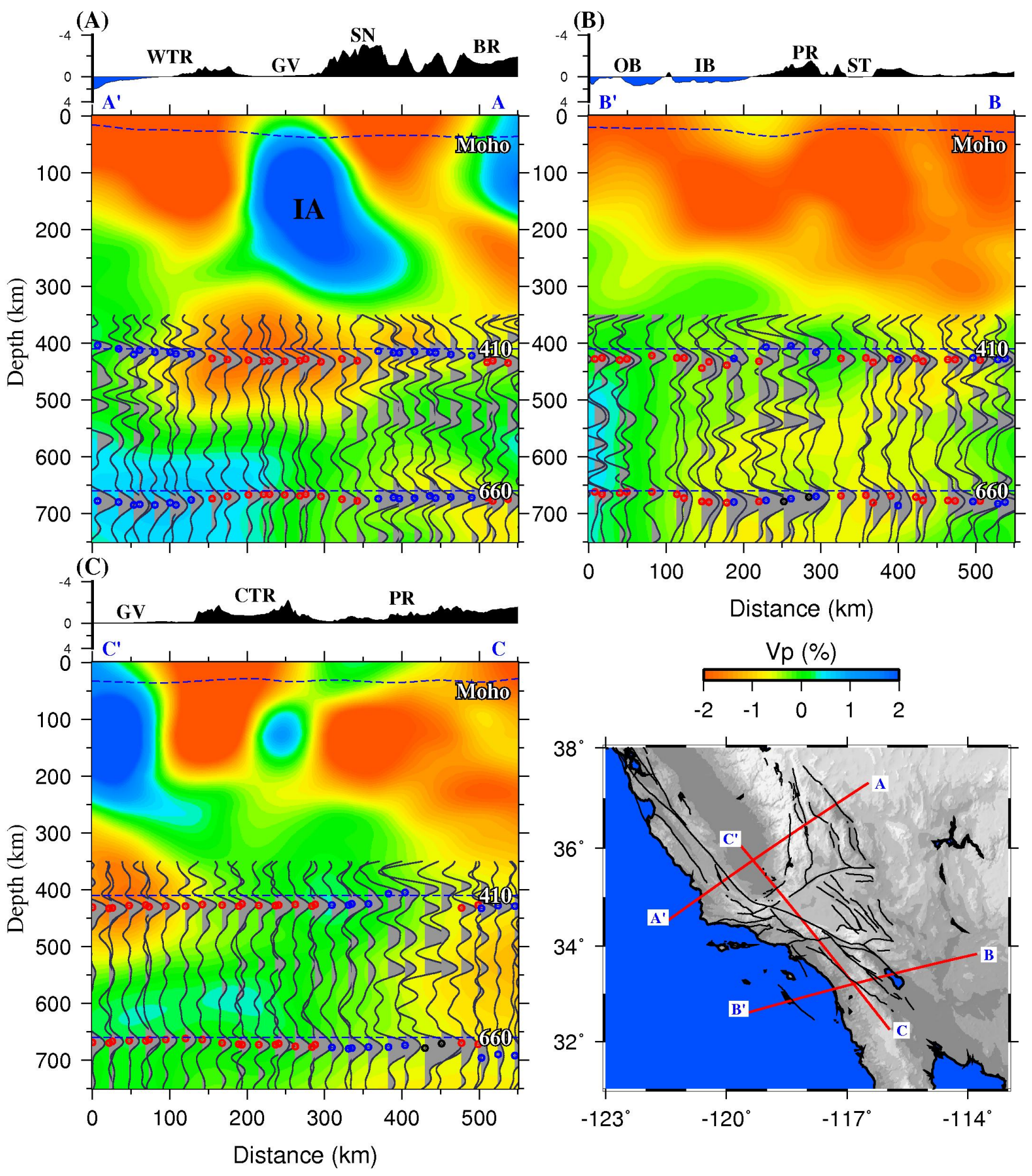

138

139 Figure DR6. Same as Figure 3 but for P-wave velocity model of Schmandt and Lin (2014). 


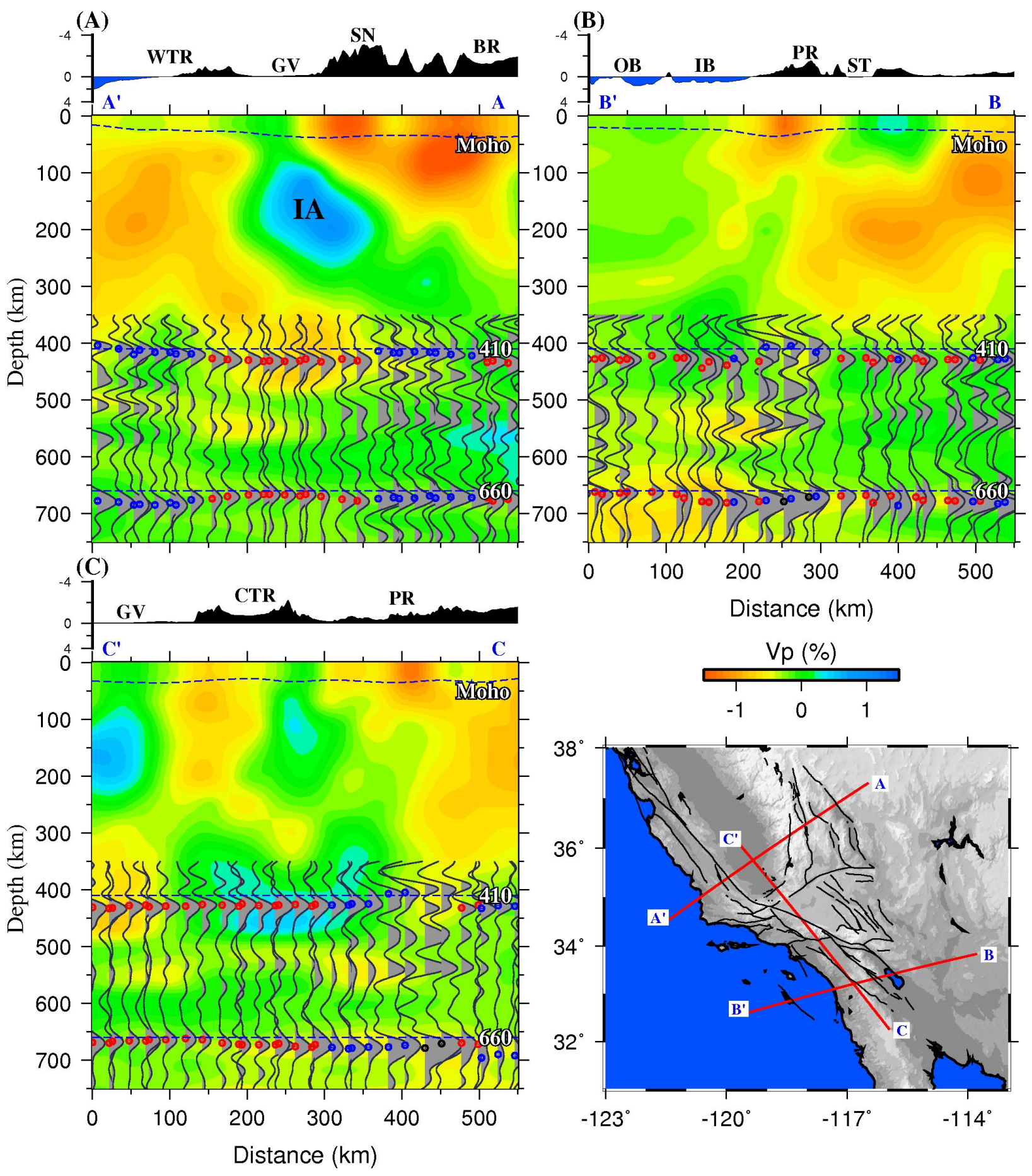

141 Figure DR7. Same as Figure 3 but for P-wave velocity model of Burdick et al. (2017). 
(A)

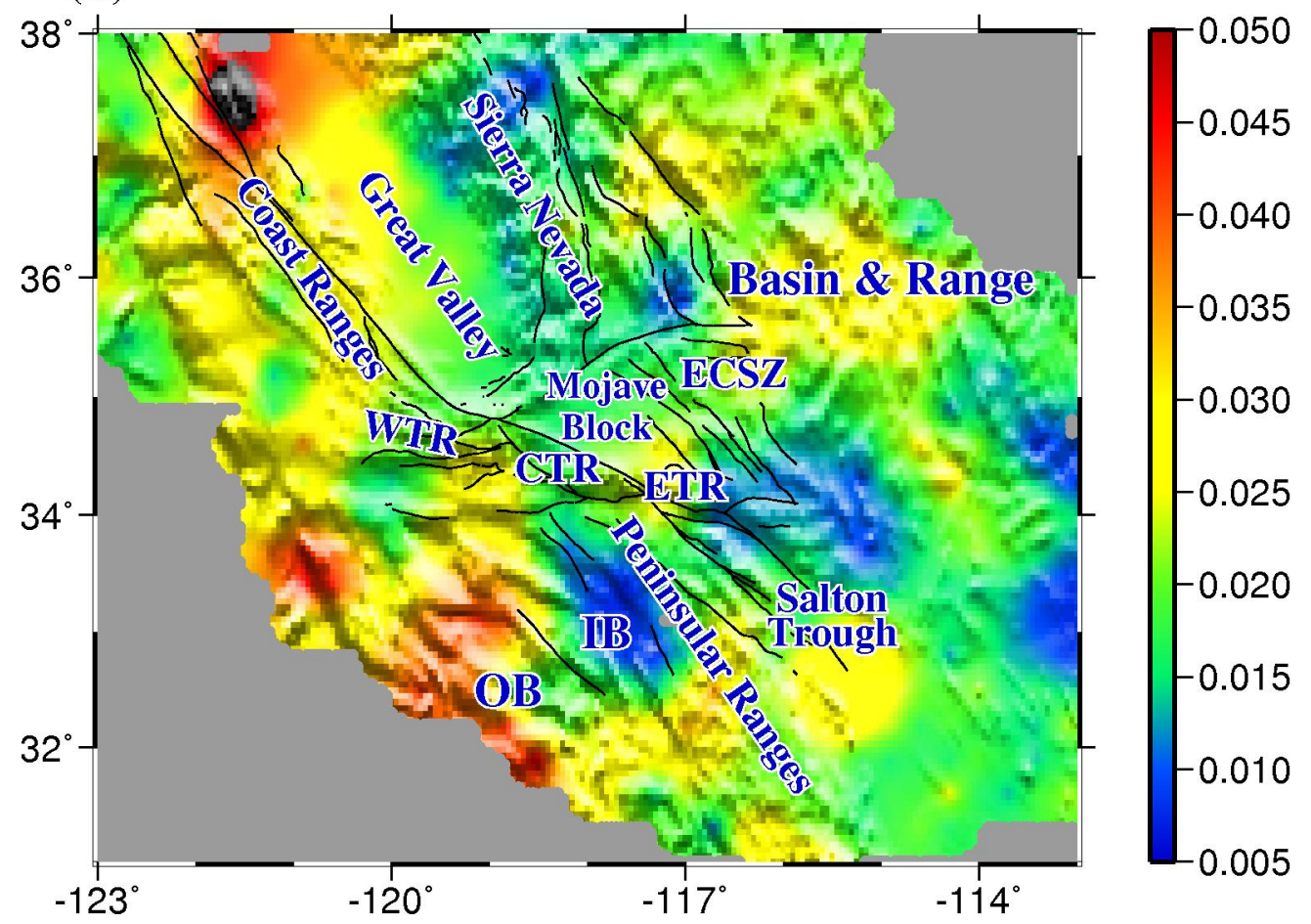

(B)

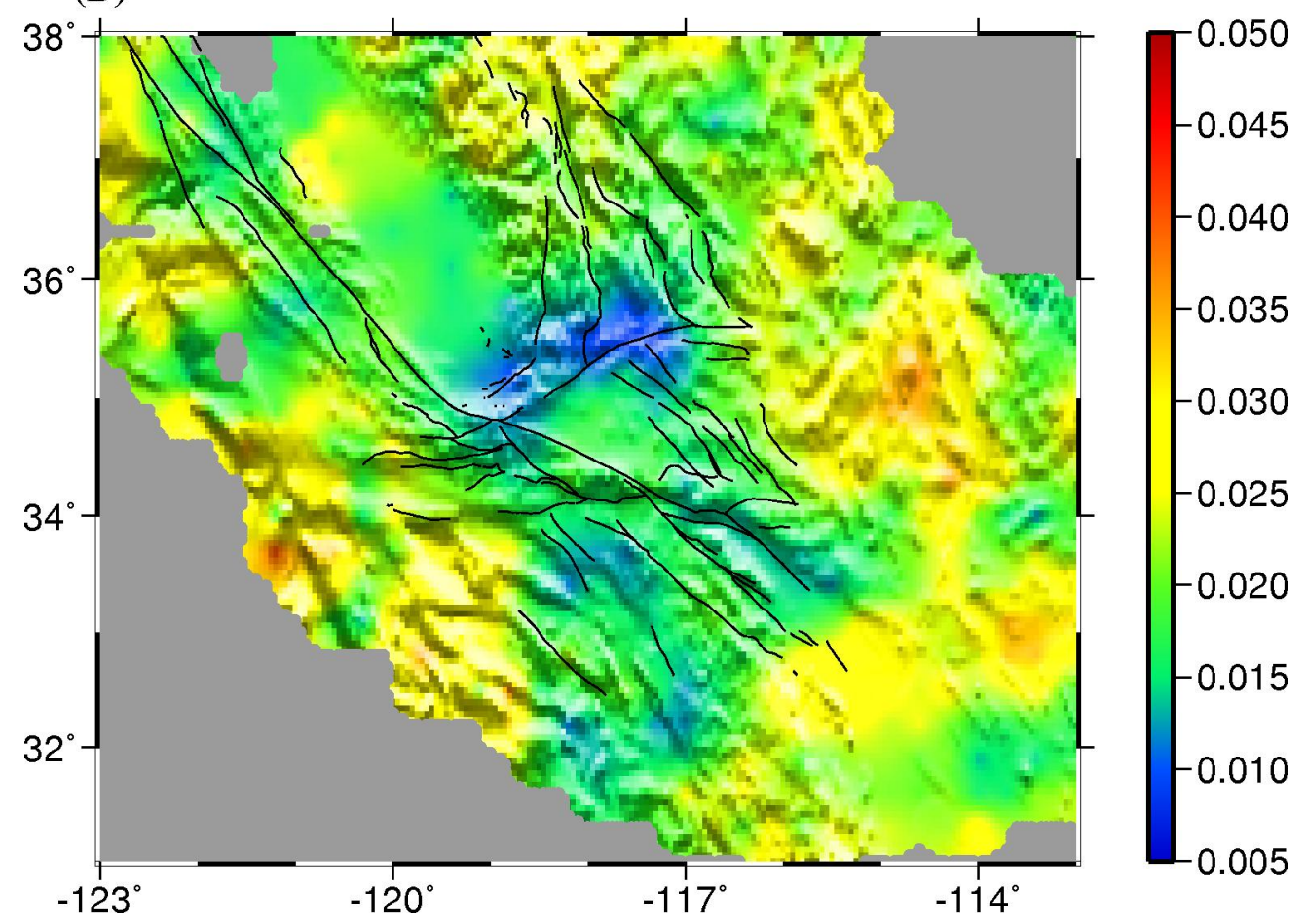

143 Figure DR8. Stacking amplitude (relative to that of the direct P-wave) for (A) d410 and (b) d660. 
144 Table DR1. Results of receiver function stacking for each of the bins. The columns are:

145 clon: longitude of the circle's center

146 clat: latitude of the circle's center

147 d410: depth of the $\mathrm{d} 410$

148 sig4: standard deviation of the d410 depth

149 d660: depth of the d660

150 sig6: standard deviation of the d660 depth

151 MTZ: Mantle Transition Zone thickness

152 sigm: standard deviation of MTZ thickness

153 NRFs: Number of receiver functions in the circle

154 Note that a value of zero is given to the depth if the Pds arrival is not well-defined.

\begin{tabular}{|c|c|c|c|c|c|c|c|c|}
\hline clon & clat & $\mathrm{d} 410$ & sig4 & $\mathrm{d} 660$ & sig6 & MTZ & sigm & NRFs \\
\hline-123 & 36.1 & 426 & 1.9 & 0 & 0 & 0 & 0 & 114 \\
\hline-123 & 36.4 & 426 & 4.5 & 0 & 0 & 0 & 0 & 108 \\
\hline-123 & 37 & 426 & 1.6 & 674 & 1.5 & 248 & 2.2 & 141 \\
\hline-123 & 37.3 & 422 & 1 & 667 & 1.2 & 244 & 1.7 & 288 \\
\hline-123 & 37.6 & 423 & 1.1 & 667 & 1.2 & 243 & 1.5 & 394 \\
\hline-123 & 37.9 & 423 & 2.3 & 668 & 2.8 & 244 & 3.2 & 284 \\
\hline-122.7 & 35.5 & 427 & 2 & 676 & 3 & 249 & 4.4 & 237 \\
\hline-122.7 & 35.8 & 424 & 0.7 & 672 & 1.2 & 247 & 1.5 & 314 \\
\hline-122.7 & 36.1 & 424 & 1.7 & 675 & 1.1 & 250 & 2.1 & 313 \\
\hline-122.7 & 36.4 & 427 & 2.1 & 0 & 0 & 0 & 0 & 205 \\
\hline-122.7 & 36.7 & 429 & 3.6 & 681 & 2.5 & 252 & 4.2 & 197 \\
\hline-122.7 & 37 & 421 & 1.6 & 671 & 1.1 & 250 & 1.9 & 290 \\
\hline-122.7 & 37.3 & 422 & 2.4 & 667 & 1 & 244 & 2.8 & 406 \\
\hline-122.7 & 37.6 & 423 & 1.3 & 669 & 0.9 & 245 & 1.6 & 488 \\
\hline-122.7 & 37.9 & 426 & 3 & 665 & 1.7 & 239 & 2.8 & 356 \\
\hline-122.4 & 35.2 & 429 & 3.3 & 677 & 3.5 & 248 & 4.8 & 173 \\
\hline-122.4 & 35.5 & 427 & 1.6 & 673 & 2.2 & 245 & 3 & 354 \\
\hline-122.4 & 35.8 & 426 & 1.1 & 673 & 1.8 & 246 & 2.2 & 413 \\
\hline-122.4 & 36.1 & 426 & 1.2 & 673 & 1.6 & 246 & 2 & 408 \\
\hline-122.4 & 36.4 & 425 & 1.3 & 0 & 0 & 0 & 0 & 323 \\
\hline-122.4 & 36.7 & 422 & 1.8 & 679 & 3.8 & 256 & 4.3 & 306 \\
\hline-122.4 & 37 & 421 & 1.4 & 670 & 1.6 & 248 & 1.7 & 403 \\
\hline-122.4 & 37.3 & 425 & 1.8 & 667 & 1.8 & 242 & 2.7 & 478 \\
\hline-122.4 & 37.6 & 426 & 1.4 & 668 & 1.7 & 241 & 2.1 & 495 \\
\hline-122.4 & 37.9 & 425 & 1.8 & 667 & 1.6 & 241 & 2.3 & 371 \\
\hline-122.1 & 34.9 & 0 & 0 & 678 & 2.5 & 0 & 0 & 150 \\
\hline-122.1 & 35.2 & 431 & 2.7 & 682 & 3.5 & 250 & 5 & 264 \\
\hline-122.1 & 35.5 & 428 & 1.1 & 675 & 2 & 246 & 2.4 & 477 \\
\hline-122.1 & 35.8 & 427 & 1.1 & 674 & 1.3 & 246 & 1.6 & 488 \\
\hline-122.1 & 36.1 & 432 & 1.5 & 676 & 1.1 & 244 & 1.9 & 575 \\
\hline-122.1 & 36.4 & 434 & 1.5 & 688 & 2.9 & 253 & 3.3 & 458 \\
\hline-122.1 & 36.7 & 430 & 1.7 & 671 & 3.1 & 241 & 3.2 & 396 \\
\hline
\end{tabular}




\begin{tabular}{|c|c|c|c|c|c|c|c|c|}
\hline-122.1 & 37 & 426 & 1.6 & 668 & 1.6 & 242 & 2.4 & 429 \\
\hline-122.1 & 37.3 & 424 & 1.2 & 666 & 1.4 & 241 & 2 & 429 \\
\hline-122.1 & 37.6 & 427 & 1.2 & 663 & 2.6 & 236 & 2.6 & 393 \\
\hline-122.1 & 37.9 & 434 & 2.8 & 0 & 0 & 0 & 0 & 200 \\
\hline-121.8 & 34.9 & 0 & 0 & 678 & 3 & 0 & 0 & 206 \\
\hline-121.8 & 35.2 & 432 & 1.9 & 0 & 0 & 0 & 0 & 292 \\
\hline-121.8 & 35.5 & 428 & 1.1 & 0 & 0 & 0 & 0 & 397 \\
\hline-121.8 & 35.8 & 431 & 1.8 & 677 & 1.3 & 246 & 1.9 & 468 \\
\hline-121.8 & 36.1 & 437 & 1.3 & 676 & 1.6 & 238 & 1.8 & 474 \\
\hline-121.8 & 36.4 & 438 & 1.2 & 679 & 3.3 & 240 & 3.6 & 450 \\
\hline-121.8 & 36.7 & 433 & 2.1 & 667 & 2.8 & 234 & 3.5 & 412 \\
\hline-121.8 & 37 & 428 & 1.5 & 666 & 1.3 & 238 & 1.9 & 337 \\
\hline-121.8 & 37.3 & 425 & 1.5 & 666 & 1.5 & 240 & 2.4 & 210 \\
\hline-121.8 & 37.6 & 427 & 1.1 & 0 & 0 & 0 & 0 & 155 \\
\hline-121.5 & 34.6 & 404 & 1.1 & 678 & 2.1 & 274 & 2.3 & 132 \\
\hline-121.5 & 34.9 & 0 & 0 & 679 & 3 & 0 & 0 & 220 \\
\hline-121.5 & 35.2 & 430 & 2 & 0 & 0 & 0 & 0 & 332 \\
\hline-121.5 & 35.5 & 434 & 1.6 & 0 & 0 & 0 & 0 & 414 \\
\hline-121.5 & 35.8 & 437 & 2.1 & 0 & 0 & 0 & 0 & 529 \\
\hline-121.5 & 36.1 & 436 & 2.2 & 684 & 1.5 & 248 & 2.8 & 533 \\
\hline-121.5 & 36.4 & 443 & 1.5 & 685 & 2.5 & 241 & 2.7 & 509 \\
\hline-121.5 & 36.7 & 438 & 1 & 673 & 1.9 & 234 & 2.2 & 421 \\
\hline-121.5 & 37 & 431 & 1.4 & 0 & 0 & 0 & 0 & 269 \\
\hline-121.5 & 37.3 & 427 & 0.9 & 0 & 0 & 0 & 0 & 162 \\
\hline-121.5 & 37.6 & 428 & 1.8 & 0 & 0 & 0 & 0 & 101 \\
\hline-121.2 & 33.7 & 430 & 1.9 & 672 & 1.1 & 242 & 2.2 & 100 \\
\hline-121.2 & 34 & 399 & 2 & 677 & 2.4 & 277 & 2.7 & 116 \\
\hline-121.2 & 34.3 & 402 & 2.8 & 684 & 2.2 & 282 & 4.2 & 210 \\
\hline-121.2 & 34.6 & 410 & 1.7 & 680 & 1.5 & 270 & 2.5 & 325 \\
\hline-121.2 & 34.9 & 420 & 2.6 & 685 & 2.3 & 265 & 3.5 & 467 \\
\hline-121.2 & 35.2 & 419 & 3.1 & 690 & 3.2 & 270 & 3.6 & 491 \\
\hline-121.2 & 35.5 & 430 & 1.2 & 690 & 3.6 & 259 & 3.8 & 559 \\
\hline-121.2 & 35.8 & 427 & 4.5 & 679 & 2.2 & 252 & 4.8 & 563 \\
\hline-121.2 & 36.1 & 440 & 1.6 & 686 & 2.1 & 245 & 2.8 & 517 \\
\hline-121.2 & 36.4 & 443 & 1.7 & 689 & 3.4 & 245 & 3.9 & 456 \\
\hline-121.2 & 36.7 & 439 & 2.6 & 678 & 2 & 238 & 3.2 & 253 \\
\hline-121.2 & 37 & 430 & 2.6 & 668 & 3.6 & 237 & 3.5 & 189 \\
\hline-121.2 & 37.3 & 421 & 1.9 & 667 & 2.8 & 245 & 3.7 & 126 \\
\hline-121.2 & 37.6 & 418 & 1.3 & 0 & 0 & 0 & 0 & 132 \\
\hline-120.9 & 33.1 & 428 & 3.6 & 0 & 0 & 0 & 0 & 141 \\
\hline-120.9 & 33.4 & 431 & 1.1 & 667 & 1.9 & 236 & 2.4 & 166 \\
\hline-120.9 & 33.7 & 432 & 1.4 & 672 & 1 & 240 & 1.5 & 186 \\
\hline-120.9 & 34 & 428 & 1.3 & 676 & 1.4 & 248 & 2.1 & 232 \\
\hline-120.9 & 34.3 & 423 & 2.9 & 680 & 2.1 & 256 & 3.7 & 322 \\
\hline-120.9 & 34.6 & 412 & 1 & 684 & 2.3 & 271 & 2.4 & 374 \\
\hline-120.9 & 34.9 & 416 & 1.4 & 685 & 2.4 & 268 & 2.6 & 512 \\
\hline-120.9 & 35.2 & 416 & 1.3 & 681 & 2.9 & 265 & 3.2 & 619 \\
\hline-120.9 & 35.5 & 428 & 1.4 & 680 & 2.1 & 251 & 2.7 & 645 \\
\hline-120.9 & 35.8 & 426 & 1.6 & 677 & 2.5 & 251 & 3.4 & 681 \\
\hline-120.9 & 36.1 & 428 & 1.9 & 0 & 0 & 0 & 0 & 557 \\
\hline-120.9 & 36.4 & 422 & 1 & 0 & 0 & 0 & 0 & 374 \\
\hline-120.9 & 36.7 & 426 & 4.4 & 0 & 0 & 0 & 0 & 230 \\
\hline-120.9 & 37 & 422 & 1.5 & 658 & 1.4 & 235 & 2.2 & 246 \\
\hline-120.9 & 37.3 & 419 & 1.9 & 657 & 2.3 & 238 & 2.9 & 280 \\
\hline
\end{tabular}




\begin{tabular}{|c|c|c|c|c|c|c|c|c|}
\hline-120.9 & 37.6 & 418 & 1.1 & 665 & 3.4 & 247 & 3.8 & 266 \\
\hline-120.9 & 37.9 & 418 & 1.4 & 664 & 3.7 & 246 & 3.9 & 234 \\
\hline-120.6 & 33.1 & 432 & 2.6 & 661 & 2.6 & 229 & 3.3 & 176 \\
\hline-120.6 & 33.4 & 433 & 1.3 & 667 & 1.6 & 234 & 2 & 234 \\
\hline-120.6 & 33.7 & 435 & 1 & 673 & 0.7 & 238 & 1 & 292 \\
\hline-120.6 & 34 & 431 & 1.6 & 676 & 1.1 & 245 & 1.6 & 285 \\
\hline-120.6 & 34.3 & 419 & 3.7 & 677 & 1.1 & 258 & 3.9 & 358 \\
\hline-120.6 & 34.6 & 415 & 1 & 687 & 3.1 & 272 & 3.2 & 461 \\
\hline-120.6 & 34.9 & 419 & 0.6 & 685 & 1.7 & 265 & 1.8 & 705 \\
\hline-120.6 & 35.2 & 419 & 0.8 & 676 & 1 & 257 & 1.3 & 909 \\
\hline-120.6 & 35.5 & 432 & 0.9 & 669 & 1.3 & 236 & 1.6 & 1194 \\
\hline-120.6 & 35.8 & 431 & 0.7 & 669 & 2 & 237 & 2.1 & 1272 \\
\hline-120.6 & 36.1 & 427 & 0.8 & 667 & 2.3 & 239 & 2.5 & 1019 \\
\hline-120.6 & 36.4 & 420 & 0.8 & 0 & 0 & 0 & 0 & 695 \\
\hline-120.6 & 36.7 & 420 & 1.1 & 659 & 2.5 & 239 & 3 & 520 \\
\hline-120.6 & 37 & 420 & 1.6 & 659 & 1.4 & 239 & 2.3 & 498 \\
\hline-120.6 & 37.3 & 419 & 1.2 & 661 & 1.4 & 241 & 1.7 & 528 \\
\hline-120.6 & 37.6 & 418 & 0.9 & 665 & 2.4 & 246 & 2.6 & 383 \\
\hline-120.6 & 37.9 & 419 & 1.1 & 666 & 3.2 & 246 & 3.5 & 322 \\
\hline-120.3 & 33.1 & 435 & 1.8 & 669 & 2 & 233 & 2.8 & 195 \\
\hline-120.3 & 33.4 & 435 & 1.4 & 672 & 1.8 & 236 & 1.7 & 300 \\
\hline-120.3 & 33.7 & 439 & 1.3 & 671 & 1.1 & 232 & 1.6 & 396 \\
\hline-120.3 & 34 & 440 & 3.8 & 673 & 1 & 233 & 3.9 & 477 \\
\hline-120.3 & 34.3 & 418 & 1.7 & 675 & 3.4 & 257 & 3.5 & 544 \\
\hline-120.3 & 34.6 & 420 & 1.2 & 689 & 3.5 & 268 & 4 & 861 \\
\hline-120.3 & 34.9 & 421 & 1.2 & 677 & 1.3 & 255 & 1.6 & 1051 \\
\hline-120.3 & 35.2 & 427 & 1.3 & 674 & 1.6 & 247 & 2.1 & 1446 \\
\hline-120.3 & 35.5 & 429 & 1 & 670 & 1 & 241 & 1.5 & 1753 \\
\hline-120.3 & 35.8 & 433 & 0.8 & 668 & 0.9 & 234 & 1.3 & 1874 \\
\hline-120.3 & 36.1 & 430 & 0.8 & 668 & 1.2 & 238 & 1.4 & 1474 \\
\hline-120.3 & 36.4 & 423 & 0.8 & 670 & 3.5 & 247 & 3.7 & 958 \\
\hline-120.3 & 36.7 & 419 & 0.8 & 659 & 1.7 & 240 & 1.9 & 820 \\
\hline-120.3 & 37 & 418 & 1 & 659 & 1.6 & 240 & 1.8 & 704 \\
\hline-120.3 & 37.3 & 420 & 1.3 & 660 & 1.4 & 239 & 2.1 & 689 \\
\hline-120.3 & 37.6 & 418 & 1 & 663 & 1.9 & 245 & 2.1 & 466 \\
\hline-120.3 & 37.9 & 419 & 1.1 & 664 & 1.4 & 244 & 1.6 & 325 \\
\hline-120 & 32.8 & 437 & 1.4 & 0 & 0 & 0 & 0 & 219 \\
\hline-120 & 33.1 & 440 & 1.7 & 664 & 1.3 & 223 & 2.2 & 378 \\
\hline-120 & 33.4 & 424 & 2.1 & 670 & 1 & 245 & 2.5 & 516 \\
\hline-120 & 33.7 & 420 & 1.1 & 671 & 1.1 & 251 & 1.5 & 704 \\
\hline-120 & 34 & 422 & 1.5 & 670 & 1 & 248 & 1.7 & 910 \\
\hline-120 & 34.3 & 419 & 1.8 & 668 & 1 & 248 & 1.9 & 995 \\
\hline-120 & 34.6 & 422 & 1.1 & 672 & 0.8 & 249 & 1.5 & 1314 \\
\hline-120 & 34.9 & 422 & 0.9 & 669 & 1.3 & 247 & 1.2 & 1605 \\
\hline-120 & 35.2 & 429 & 0.8 & 669 & 0.9 & 240 & 1.4 & 2172 \\
\hline-120 & 35.5 & 430 & 0.8 & 667 & 0.9 & 237 & 1.4 & 2601 \\
\hline-120 & 35.8 & 432 & 0.7 & 666 & 0.8 & 234 & 1.1 & 2387 \\
\hline-120 & 36.1 & 430 & 0.9 & 667 & 0.9 & 236 & 1.4 & 1764 \\
\hline-120 & 36.4 & 422 & 0.8 & 672 & 2.4 & 249 & 2.5 & 1197 \\
\hline-120 & 36.7 & 418 & 0.9 & 661 & 1.6 & 243 & 1.8 & 964 \\
\hline-120 & 37 & 418 & 1 & 659 & 2.3 & 241 & 2.3 & 767 \\
\hline-120 & 37.3 & 421 & 1.5 & 662 & 1.2 & 241 & 2.1 & 697 \\
\hline-120 & 37.6 & 421 & 1.2 & 667 & 1.3 & 246 & 1.9 & 442 \\
\hline-120 & 37.9 & 420 & 0.7 & 667 & 1.4 & 247 & 1.5 & 293 \\
\hline
\end{tabular}




\begin{tabular}{|c|c|c|c|c|c|c|c|c|}
\hline-119.7 & 32.5 & 434 & 1.6 & 662 & 2.7 & 227 & 2.7 & 201 \\
\hline-119.7 & 32.8 & 427 & 1 & 664 & 0.9 & 237 & 1.4 & 450 \\
\hline-119.7 & 33.1 & 424 & 0.6 & 667 & 0.7 & 242 & 0.9 & 914 \\
\hline-119.7 & 33.4 & 423 & 0.6 & 669 & 0.8 & 246 & 1 & 1405 \\
\hline-119.7 & 33.7 & 424 & 1 & 671 & 0.7 & 247 & 1.3 & 1630 \\
\hline-119.7 & 34 & 423 & 0.7 & 672 & 0.6 & 249 & 0.9 & 1663 \\
\hline-119.7 & 34.3 & 423 & 0.9 & 670 & 0.9 & 246 & 1.3 & 1790 \\
\hline-119.7 & 34.6 & 424 & 1.2 & 671 & 1 & 246 & 1.5 & 2011 \\
\hline-119.7 & 34.9 & 425 & 1 & 671 & 0.9 & 245 & 1.4 & 2418 \\
\hline-119.7 & 35.2 & 427 & 0.8 & 667 & 0.8 & 239 & 1.1 & 2884 \\
\hline-119.7 & 35.5 & 431 & 0.9 & 666 & 1 & 234 & 1.4 & 2838 \\
\hline-119.7 & 35.8 & 432 & 0.7 & 667 & 0.7 & 234 & 0.9 & 2488 \\
\hline-119.7 & 36.1 & 431 & 0.9 & 669 & 1.3 & 237 & 1.4 & 1748 \\
\hline-119.7 & 36.4 & 425 & 2.4 & 674 & 4.1 & 249 & 4.5 & 1065 \\
\hline-119.7 & 36.7 & 416 & 1.2 & 0 & 0 & 0 & 0 & 733 \\
\hline-119.7 & 37 & 415 & 2 & 663 & 1.1 & 248 & 2.6 & 635 \\
\hline-119.7 & 37.3 & 420 & 0.9 & 665 & 1.1 & 244 & 1.5 & 573 \\
\hline-119.7 & 37.6 & 422 & 1.1 & 667 & 1.4 & 245 & 1.8 & 343 \\
\hline-119.7 & 37.9 & 421 & 1.4 & 668 & 1.8 & 246 & 1.9 & 229 \\
\hline-119.4 & 32.5 & 428 & 2.8 & 662 & 1.8 & 233 & 3.2 & 286 \\
\hline-119.4 & 32.8 & 426 & 0.9 & 666 & 0.9 & 239 & 1.4 & 569 \\
\hline-119.4 & 33.1 & 425 & 0.8 & 668 & 0.7 & 243 & 1.1 & 1198 \\
\hline-119.4 & 33.4 & 425 & 0.5 & 670 & 0.4 & 245 & 0.7 & 1946 \\
\hline-119.4 & 33.7 & 425 & 0.5 & 671 & 0.6 & 246 & 0.6 & 2401 \\
\hline-119.4 & 34 & 425 & 0.6 & 672 & 0.6 & 246 & 0.8 & 2296 \\
\hline-119.4 & 34.3 & 424 & 0.6 & 672 & 1 & 248 & 1.1 & 2376 \\
\hline-119.4 & 34.6 & 422 & 0.8 & 669 & 1.3 & 246 & 1.4 & 2545 \\
\hline-119.4 & 34.9 & 426 & 1.2 & 669 & 1 & 243 & 1.7 & 2923 \\
\hline-119.4 & 35.2 & 427 & 0.6 & 664 & 0.9 & 236 & 1.1 & 3081 \\
\hline-119.4 & 35.5 & 430 & 0.8 & 664 & 0.7 & 234 & 1 & 2746 \\
\hline-119.4 & 35.8 & 428 & 0.6 & 666 & 0.9 & 237 & 1 & 2232 \\
\hline-119.4 & 36.1 & 433 & 1.1 & 670 & 1.7 & 236 & 2.2 & 1302 \\
\hline-119.4 & 36.4 & 434 & 2.9 & 677 & 2.4 & 243 & 3.6 & 741 \\
\hline-119.4 & 36.7 & 414 & 3.4 & 663 & 3.7 & 248 & 5.2 & 541 \\
\hline-119.4 & 37 & 420 & 3.3 & 664 & 1.7 & 243 & 3.4 & 620 \\
\hline-119.4 & 37.3 & 422 & 1.4 & 665 & 0.6 & 243 & 1.6 & 538 \\
\hline-119.4 & 37.6 & 422 & 1.1 & 667 & 0.8 & 244 & 1.3 & 395 \\
\hline-119.4 & 37.9 & 420 & 1 & 666 & 1.4 & 245 & 1.6 & 222 \\
\hline-119.1 & 32.5 & 430 & 1.6 & 664 & 1.8 & 233 & 2.3 & 217 \\
\hline-119.1 & 32.8 & 428 & 0.9 & 662 & 1.1 & 234 & 1.3 & 668 \\
\hline-119.1 & 33.1 & 426 & 0.6 & 667 & 0.8 & 240 & 0.9 & 1470 \\
\hline-119.1 & 33.4 & 425 & 0.5 & 668 & 0.5 & 243 & 0.8 & 2017 \\
\hline-119.1 & 33.7 & 425 & 0.6 & 671 & 0.7 & 245 & 0.9 & 2449 \\
\hline-119.1 & 34 & 423 & 0.6 & 672 & 0.6 & 248 & 0.8 & 2445 \\
\hline-119.1 & 34.3 & 421 & 0.7 & 674 & 1.1 & 252 & 1.3 & 2290 \\
\hline-119.1 & 34.6 & 423 & 1.1 & 672 & 1 & 249 & 1.4 & 2559 \\
\hline-119.1 & 34.9 & 429 & 2.8 & 666 & 1.4 & 236 & 2.7 & 2850 \\
\hline-119.1 & 35.2 & 429 & 1.2 & 662 & 1.5 & 233 & 1.7 & 3089 \\
\hline-119.1 & 35.5 & 429 & 0.8 & 664 & 1 & 235 & 1.3 & 2713 \\
\hline-119.1 & 35.8 & 427 & 1 & 666 & 1.6 & 239 & 1.7 & 1896 \\
\hline-119.1 & 36.1 & 428 & 0.8 & 675 & 1.1 & 246 & 1.5 & 1209 \\
\hline-119.1 & 36.4 & 431 & 2.1 & 678 & 1.3 & 246 & 2.4 & 791 \\
\hline-119.1 & 36.7 & 420 & 1.8 & 666 & 1.7 & 246 & 2.9 & 666 \\
\hline-119.1 & 37 & 420 & 1.6 & 665 & 1.2 & 244 & 1.9 & 745 \\
\hline
\end{tabular}




\begin{tabular}{|c|c|c|c|c|c|c|c|c|}
\hline-119.1 & 37.3 & 423 & 1.5 & 667 & 0.9 & 243 & 1.6 & 674 \\
\hline-119.1 & 37.6 & 420 & 1 & 667 & 0.9 & 246 & 1.3 & 511 \\
\hline-119.1 & 37.9 & 422 & 1.2 & 666 & 1.5 & 243 & 2.3 & 350 \\
\hline-118.8 & 32.2 & 429 & 0.7 & 664 & 1.6 & 234 & 1.8 & 180 \\
\hline-118.8 & 32.5 & 424 & 1 & 660 & 0.8 & 236 & 1.3 & 331 \\
\hline-118.8 & 32.8 & 422 & 0.8 & 662 & 1.2 & 240 & 1.3 & 655 \\
\hline-118.8 & 33.1 & 427 & 0.7 & 665 & 0.9 & 238 & 1.1 & 1166 \\
\hline-118.8 & 33.4 & 428 & 0.8 & 670 & 1.3 & 241 & 1.7 & 1643 \\
\hline-118.8 & 33.7 & 426 & 0.7 & 670 & 1.1 & 243 & 1.2 & 2036 \\
\hline-118.8 & 34 & 424 & 0.5 & 670 & 1 & 245 & 1.2 & 2322 \\
\hline-118.8 & 34.3 & 426 & 1 & 671 & 1.2 & 244 & 1.4 & 2359 \\
\hline-118.8 & 34.6 & 425 & 0.8 & 673 & 1 & 248 & 1.2 & 2626 \\
\hline-118.8 & 34.9 & 427 & 0.8 & 670 & 0.8 & 243 & 1 & 2825 \\
\hline-118.8 & 35.2 & 426 & 0.8 & 664 & 1.3 & 238 & 1.7 & 3332 \\
\hline-118.8 & 35.5 & 427 & 0.9 & 660 & 1.3 & 233 & 1.6 & 2952 \\
\hline-118.8 & 35.8 & 426 & 1.4 & 666 & 2.8 & 240 & 3.1 & 2071 \\
\hline-118.8 & 36.1 & 421 & 2.7 & 675 & 0.6 & 254 & 3 & 1487 \\
\hline-118.8 & 36.4 & 414 & 2.5 & 674 & 1 & 260 & 2.6 & 1371 \\
\hline-118.8 & 36.7 & 417 & 1.3 & 672 & 0.9 & 254 & 1.6 & 1235 \\
\hline-118.8 & 37 & 418 & 1.4 & 667 & 0.9 & 248 & 1.5 & 1027 \\
\hline-118.8 & 37.3 & 419 & 1.5 & 665 & 0.9 & 246 & 1.9 & 817 \\
\hline-118.8 & 37.6 & 425 & 2.2 & 668 & 1 & 243 & 2.3 & 694 \\
\hline-118.8 & 37.9 & 425 & 1.6 & 668 & 1.8 & 242 & 2.9 & 466 \\
\hline-118.5 & 31.9 & 431 & 0.7 & 674 & 2.1 & 242 & 2.2 & 320 \\
\hline-118.5 & 32.2 & 431 & 0.5 & 671 & 1.7 & 240 & 1.7 & 709 \\
\hline-118.5 & 32.5 & 428 & 1 & 666 & 1.2 & 238 & 1.6 & 1289 \\
\hline-118.5 & 32.8 & 426 & 0.9 & 666 & 1.4 & 240 & 1.8 & 1659 \\
\hline-118.5 & 33.1 & 426 & 0.7 & 673 & 2.6 & 247 & 2.8 & 2002 \\
\hline-118.5 & 33.4 & 430 & 0.8 & 672 & 2.4 & 241 & 2.7 & 2449 \\
\hline-118.5 & 33.7 & 427 & 0.7 & 668 & 1 & 240 & 1.4 & 2847 \\
\hline-118.5 & 34 & 427 & 1 & 670 & 2.1 & 243 & 2.4 & 2879 \\
\hline-118.5 & 34.3 & 427 & 1.1 & 671 & 1.5 & 243 & 1.5 & 2889 \\
\hline-118.5 & 34.6 & 428 & 0.7 & 673 & 0.8 & 244 & 1.2 & 3865 \\
\hline-118.5 & 34.9 & 429 & 0.7 & 672 & 0.5 & 243 & 0.9 & 3995 \\
\hline-118.5 & 35.2 & 426 & 0.6 & 668 & 0.7 & 242 & 0.9 & 3581 \\
\hline-118.5 & 35.5 & 426 & 0.7 & 661 & 1.6 & 234 & 1.9 & 2821 \\
\hline-118.5 & 35.8 & 424 & 1.4 & 660 & 2 & 236 & 2.4 & 2415 \\
\hline-118.5 & 36.1 & 415 & 1.2 & 676 & 0.9 & 261 & 1.3 & 1720 \\
\hline-118.5 & 36.4 & 417 & 1.2 & 674 & 0.9 & 256 & 1.6 & 1594 \\
\hline-118.5 & 36.7 & 415 & 1.2 & 673 & 1 & 258 & 1.6 & 1462 \\
\hline-118.5 & 37 & 416 & 1.4 & 669 & 1.4 & 252 & 2.2 & 1141 \\
\hline-118.5 & 37.3 & 418 & 1.4 & 666 & 1.5 & 247 & 2 & 1033 \\
\hline-118.5 & 37.6 & 417 & 4 & 669 & 1.3 & 252 & 4.2 & 716 \\
\hline-118.5 & 37.9 & 428 & 2.5 & 670 & 1.5 & 242 & 2.9 & 458 \\
\hline-118.2 & 31.9 & 433 & 0.8 & 674 & 2.2 & 241 & 2.3 & 567 \\
\hline-118.2 & 32.2 & 434 & 1 & 679 & 3.1 & 245 & 3 & 1174 \\
\hline-118.2 & 32.5 & 433 & 1.2 & 678 & 1.6 & 245 & 1.8 & 2220 \\
\hline-118.2 & 32.8 & 444 & 4.2 & 679 & 1.1 & 234 & 4.3 & 3132 \\
\hline-118.2 & 33.1 & 433 & 3.1 & 680 & 1.9 & 247 & 3.4 & 4269 \\
\hline-118.2 & 33.4 & 429 & 0.9 & 678 & 1.5 & 248 & 1.7 & 4181 \\
\hline-118.2 & 33.7 & 429 & 1.2 & 676 & 1 & 246 & 1.7 & 3881 \\
\hline-118.2 & 34 & 426 & 1 & 673 & 1.6 & 246 & 1.9 & 3973 \\
\hline-118.2 & 34.3 & 426 & 0.6 & 676 & 0.7 & 249 & 1 & 5218 \\
\hline-118.2 & 34.6 & 428 & 0.9 & 673 & 0.6 & 245 & 1.1 & 6316 \\
\hline
\end{tabular}




\begin{tabular}{|c|c|c|c|c|c|c|c|c|}
\hline-118.2 & 34.9 & 428 & 0.8 & 673 & 0.4 & 245 & 1 & 5821 \\
\hline-118.2 & 35.2 & 426 & 0.6 & 671 & 0.4 & 245 & 0.8 & 4751 \\
\hline-118.2 & 35.5 & 428 & 0.9 & 665 & 1.9 & 237 & 2.3 & 2973 \\
\hline-118.2 & 35.8 & 419 & 1.5 & 661 & 2.1 & 242 & 2.6 & 2574 \\
\hline-118.2 & 36.1 & 421 & 2.8 & 675 & 1 & 254 & 3.1 & 2127 \\
\hline-118.2 & 36.4 & 420 & 0.8 & 676 & 0.8 & 255 & 1.3 & 1796 \\
\hline-118.2 & 36.7 & 417 & 1.5 & 673 & 0.9 & 256 & 1.7 & 1432 \\
\hline-118.2 & 37 & 420 & 0.8 & 671 & 1 & 251 & 1.1 & 1313 \\
\hline-118.2 & 37.3 & 0 & 0 & 670 & 1.5 & 0 & 0 & 1004 \\
\hline-118.2 & 37.6 & 433 & 1.5 & 670 & 1.6 & 236 & 2.4 & 672 \\
\hline-118.2 & 37.9 & 426 & 2 & 670 & 1.4 & 244 & 2.5 & 516 \\
\hline-117.9 & 31.6 & 440 & 1.7 & 667 & 4.1 & 227 & 4.7 & 158 \\
\hline-117.9 & 31.9 & 434 & 1.1 & 682 & 1.7 & 248 & 1.9 & 779 \\
\hline-117.9 & 32.2 & 436 & 0.8 & 683 & 1.3 & 247 & 1.6 & 1577 \\
\hline-117.9 & 32.5 & 434 & 1 & 684 & 1.5 & 249 & 1.8 & 3956 \\
\hline-117.9 & 32.8 & 439 & 1.6 & 681 & 1.6 & 242 & 2.4 & 5954 \\
\hline-117.9 & 33.1 & 427 & 1.2 & 680 & 0.8 & 253 & 1.4 & 7605 \\
\hline-117.9 & 33.4 & 424 & 1 & 679 & 0.9 & 255 & 1.4 & 6371 \\
\hline-117.9 & 33.7 & 425 & 0.8 & 679 & 0.8 & 253 & 1.1 & 5553 \\
\hline-117.9 & 34 & 427 & 0.5 & 678 & 0.8 & 251 & 1 & 6014 \\
\hline-117.9 & 34.3 & 429 & 0.3 & 677 & 0.5 & 248 & 0.7 & 7814 \\
\hline-117.9 & 34.6 & 429 & 0.5 & 676 & 0.5 & 247 & 0.6 & 8378 \\
\hline-117.9 & 34.9 & 428 & 0.8 & 674 & 0.5 & 246 & 1 & 6585 \\
\hline-117.9 & 35.2 & 429 & 0.7 & 672 & 0.7 & 243 & 0.9 & 4991 \\
\hline-117.9 & 35.5 & 425 & 1.3 & 661 & 2.9 & 235 & 3.3 & 2936 \\
\hline-117.9 & 35.8 & 420 & 1.5 & 661 & 1.9 & 241 & 2.4 & 2020 \\
\hline-117.9 & 36.1 & 423 & 2.2 & 675 & 1.4 & 252 & 2.4 & 1758 \\
\hline-117.9 & 36.4 & 422 & 0.9 & 677 & 0.8 & 254 & 1.3 & 1642 \\
\hline-117.9 & 36.7 & 424 & 0.8 & 676 & 1 & 252 & 1.4 & 1426 \\
\hline-117.9 & 37 & 422 & 1.3 & 672 & 0.8 & 250 & 1.5 & 960 \\
\hline-117.9 & 37.3 & 434 & 1.6 & 672 & 1.3 & 238 & 2.3 & 697 \\
\hline-117.9 & 37.6 & 431 & 1.3 & 673 & 1.2 & 241 & 2 & 678 \\
\hline-117.9 & 37.9 & 427 & 2 & 671 & 0.9 & 244 & 2.3 & 553 \\
\hline-117.6 & 31.6 & 443 & 1.4 & 669 & 3.7 & 226 & 4 & 241 \\
\hline-117.6 & 31.9 & 435 & 1.4 & 687 & 2.2 & 252 & 2.7 & 740 \\
\hline-117.6 & 32.2 & 438 & 0.8 & 688 & 1.2 & 250 & 1.5 & 1654 \\
\hline-117.6 & 32.5 & 439 & 0.6 & 689 & 0.8 & 250 & 1.2 & 4324 \\
\hline-117.6 & 32.8 & 437 & 0.9 & 682 & 1 & 244 & 1.3 & 6373 \\
\hline-117.6 & 33.1 & 432 & 0.8 & 679 & 0.8 & 247 & 1.2 & 8166 \\
\hline-117.6 & 33.4 & 407 & 1.7 & 677 & 0.9 & 269 & 1.9 & 6850 \\
\hline-117.6 & 33.7 & 425 & 0.8 & 677 & 0.6 & 252 & 0.9 & 5982 \\
\hline-117.6 & 34 & 427 & 0.5 & 680 & 0.5 & 252 & 0.7 & 5980 \\
\hline-117.6 & 34.3 & 429 & 0.5 & 679 & 0.6 & 250 & 0.8 & 7226 \\
\hline-117.6 & 34.6 & 428 & 0.5 & 677 & 0.5 & 248 & 0.8 & 7999 \\
\hline-117.6 & 34.9 & 430 & 0.6 & 675 & 0.4 & 245 & 0.7 & 6554 \\
\hline-117.6 & 35.2 & 432 & 1.2 & 675 & 0.6 & 243 & 1.3 & 4135 \\
\hline-117.6 & 35.5 & 439 & 1.1 & 674 & 1.5 & 235 & 1.8 & 2495 \\
\hline-117.6 & 35.8 & 427 & 2 & 671 & 1.6 & 243 & 2.8 & 2004 \\
\hline-117.6 & 36.1 & 430 & 1 & 674 & 2.1 & 243 & 2.1 & 1629 \\
\hline-117.6 & 36.4 & 430 & 0.5 & 678 & 1.1 & 248 & 1.3 & 1528 \\
\hline-117.6 & 36.7 & 432 & 0.7 & 677 & 1.1 & 245 & 1.4 & 1063 \\
\hline-117.6 & 37 & 431 & 0.6 & 678 & 1.3 & 246 & 1.5 & 866 \\
\hline-117.6 & 37.3 & 435 & 2.8 & 675 & 1.8 & 240 & 3.5 & 728 \\
\hline-117.6 & 37.6 & 433 & 2 & 679 & 2.5 & 245 & 3.5 & 660 \\
\hline
\end{tabular}




\begin{tabular}{|c|c|c|c|c|c|c|c|c|}
\hline-117.6 & 37.9 & 426 & 2.2 & 680 & 1.9 & 254 & 3.2 & 452 \\
\hline-117.3 & 31.6 & 442 & 3.3 & 676 & 3.3 & 233 & 4.4 & 329 \\
\hline-117.3 & 31.9 & 433 & 2.5 & 687 & 2 & 253 & 3.4 & 744 \\
\hline-117.3 & 32.2 & 438 & 0.9 & 689 & 2.4 & 251 & 2.6 & 1479 \\
\hline-117.3 & 32.5 & 436 & 1.1 & 694 & 1.3 & 258 & 1.8 & 3617 \\
\hline-117.3 & 32.8 & 436 & 0.8 & 684 & 2 & 248 & 2.2 & 5584 \\
\hline-117.3 & 33.1 & 0 & 0 & 679 & 1.2 & 0 & 0 & 7405 \\
\hline-117.3 & 33.4 & 405 & 0.7 & 674 & 0.9 & 268 & 1.2 & 6011 \\
\hline-117.3 & 33.7 & 421 & 1.5 & 679 & 1.1 & 257 & 1.6 & 4854 \\
\hline-117.3 & 34 & 429 & 0.6 & 681 & 0.8 & 251 & 1 & 4676 \\
\hline-117.3 & 34.3 & 428 & 0.5 & 680 & 0.5 & 252 & 0.8 & 5735 \\
\hline-117.3 & 34.6 & 429 & 0.5 & 680 & 0.5 & 250 & 0.8 & 6162 \\
\hline-117.3 & 34.9 & 430 & 0.6 & 677 & 0.8 & 246 & 1 & 4826 \\
\hline-117.3 & 35.2 & 434 & 0.6 & 679 & 1.2 & 245 & 1.4 & 2636 \\
\hline-117.3 & 35.5 & 440 & 1.4 & 677 & 1.1 & 237 & 1.7 & 2027 \\
\hline-117.3 & 35.8 & 431 & 2.6 & 677 & 1.4 & 245 & 3.2 & 1891 \\
\hline-117.3 & 36.1 & 429 & 2.9 & 681 & 1.6 & 251 & 3.5 & 1648 \\
\hline-117.3 & 36.4 & 433 & 0.7 & 677 & 1.5 & 243 & 1.6 & 1257 \\
\hline-117.3 & 36.7 & 434 & 0.6 & 681 & 1.5 & 247 & 1.5 & 941 \\
\hline-117.3 & 37 & 433 & 0.8 & 676 & 1 & 243 & 1.3 & 786 \\
\hline-117.3 & 37.3 & 433 & 1.4 & 684 & 1.9 & 250 & 2.2 & 714 \\
\hline-117.3 & 37.6 & 433 & 3.3 & 686 & 2 & 253 & 4 & 435 \\
\hline-117.3 & 37.9 & 427 & 2.7 & 684 & 1.7 & 256 & 3.2 & 423 \\
\hline-117 & 31.3 & 439 & 1.9 & 679 & 2.4 & 239 & 2.6 & 100 \\
\hline-117 & 31.6 & 425 & 2.4 & 681 & 1.8 & 256 & 3 & 336 \\
\hline-117 & 31.9 & 429 & 1.4 & 690 & 2.5 & 261 & 2.9 & 782 \\
\hline-117 & 32.2 & 429 & 0.8 & 691 & 2 & 262 & 2.1 & 1252 \\
\hline-117 & 32.5 & 433 & 0.7 & 696 & 2.2 & 263 & 2.3 & 2353 \\
\hline-117 & 32.8 & 432 & 0.7 & 670 & 1.3 & 237 & 1.4 & 4057 \\
\hline-117 & 33.1 & 0 & 0 & 671 & 1.2 & 0 & 0 & 5283 \\
\hline-117 & 33.4 & 416 & 2.1 & 670 & 1 & 254 & 2 & 4374 \\
\hline-117 & 33.7 & 429 & 1.1 & 677 & 1.5 & 247 & 2.3 & 3212 \\
\hline-117 & 34 & 430 & 0.6 & 683 & 1.9 & 252 & 1.9 & 3145 \\
\hline-117 & 34.3 & 431 & 0.5 & 683 & 1.2 & 251 & 1.4 & 3957 \\
\hline-117 & 34.6 & 431 & 0.2 & 681 & 1.3 & 250 & 1.3 & 3539 \\
\hline-117 & 34.9 & 433 & 0.6 & 680 & 0.7 & 247 & 0.9 & 2580 \\
\hline-117 & 35.2 & 435 & 0.8 & 679 & 0.8 & 244 & 1 & 2259 \\
\hline-117 & 35.5 & 435 & 0.8 & 680 & 0.9 & 244 & 1.3 & 2222 \\
\hline-117 & 35.8 & 430 & 3.6 & 679 & 1.1 & 248 & 3.6 & 1732 \\
\hline-117 & 36.1 & 431 & 0.8 & 681 & 2.3 & 249 & 2.1 & 1319 \\
\hline-117 & 36.4 & 433 & 0.7 & 679 & 1.2 & 245 & 1.3 & 1168 \\
\hline-117 & 36.7 & 434 & 0.8 & 680 & 1.9 & 245 & 2.2 & 892 \\
\hline-117 & 37 & 434 & 0.8 & 677 & 1.6 & 242 & 1.9 & 657 \\
\hline-117 & 37.3 & 430 & 1.4 & 687 & 4.3 & 257 & 4.9 & 502 \\
\hline-117 & 37.6 & 429 & 1.5 & 686 & 2.9 & 256 & 3.1 & 471 \\
\hline-117 & 37.9 & 425 & 2.5 & 688 & 1.5 & 263 & 2.9 & 354 \\
\hline-116.7 & 31.3 & 437 & 3.3 & 683 & 2.1 & 245 & 4.1 & 156 \\
\hline-116.7 & 31.6 & 438 & 1.4 & 684 & 1.5 & 246 & 1.7 & 587 \\
\hline-116.7 & 31.9 & 434 & 1.2 & 692 & 1.7 & 257 & 2.2 & 974 \\
\hline-116.7 & 32.2 & 430 & 0.6 & 691 & 1.7 & 260 & 1.9 & 1408 \\
\hline-116.7 & 32.5 & 428 & 0.6 & 690 & 3.3 & 262 & 3.4 & 1589 \\
\hline-116.7 & 32.8 & 427 & 0.5 & 672 & 1 & 244 & 1.1 & 2414 \\
\hline-116.7 & 33.1 & 426 & 0.7 & 670 & 0.5 & 243 & 0.9 & 3021 \\
\hline-116.7 & 33.4 & 427 & 0.8 & 669 & 1 & 241 & 1.4 & 3425 \\
\hline
\end{tabular}




\begin{tabular}{|c|c|c|c|c|c|c|c|c|}
\hline-116.7 & 33.7 & 433 & 1.3 & 681 & 4.1 & 247 & 4.1 & 2826 \\
\hline-116.7 & 34 & 444 & 1 & 681 & 3.9 & 236 & 3.9 & 2354 \\
\hline-116.7 & 34.3 & 435 & 4 & 683 & 1.2 & 248 & 4.3 & 2448 \\
\hline-116.7 & 34.6 & 433 & 0.9 & 680 & 1.4 & 246 & 1.4 & 2135 \\
\hline-116.7 & 34.9 & 432 & 0.8 & 680 & 0.7 & 248 & 1.1 & 2245 \\
\hline-116.7 & 35.2 & 433 & 0.6 & 681 & 0.9 & 248 & 1.1 & 2109 \\
\hline-116.7 & 35.5 & 432 & 0.5 & 678 & 1.1 & 245 & 1.1 & 1995 \\
\hline-116.7 & 35.8 & 430 & 0.7 & 674 & 1 & 244 & 1 & 1672 \\
\hline-116.7 & 36.1 & 433 & 1.1 & 679 & 1.5 & 246 & 1.8 & 1272 \\
\hline-116.7 & 36.4 & 433 & 1 & 681 & 1.6 & 247 & 1.8 & 1029 \\
\hline-116.7 & 36.7 & 433 & 0.7 & 677 & 1.3 & 244 & 1.4 & 727 \\
\hline-116.7 & 37 & 430 & 1 & 673 & 1.2 & 243 & 1.6 & 471 \\
\hline-116.7 & 37.3 & 434 & 2.3 & 674 & 3.3 & 239 & 4 & 515 \\
\hline-116.7 & 37.6 & 432 & 2.5 & 687 & 2.4 & 254 & 3.5 & 450 \\
\hline-116.7 & 37.9 & 428 & 2.5 & 687 & 2.1 & 258 & 3.8 & 319 \\
\hline-116.4 & 31.3 & 437 & 1.7 & 686 & 2.5 & 249 & 3.3 & 239 \\
\hline-116.4 & 31.6 & 438 & 1 & 687 & 1.7 & 248 & 2 & 766 \\
\hline-116.4 & 31.9 & 436 & 1.3 & 690 & 1 & 254 & 1.6 & 1085 \\
\hline-116.4 & 32.2 & 433 & 0.9 & 690 & 1.5 & 257 & 1.8 & 1828 \\
\hline-116.4 & 32.5 & 429 & 1.3 & 692 & 1.3 & 262 & 1.7 & 2507 \\
\hline-116.4 & 32.8 & 427 & 0.5 & 679 & 3.5 & 252 & 3.5 & 2875 \\
\hline-116.4 & 33.1 & 427 & 0.6 & 672 & 1 & 244 & 1.2 & 2741 \\
\hline-116.4 & 33.4 & 426 & 0.9 & 668 & 1 & 241 & 1.3 & 2969 \\
\hline-116.4 & 33.7 & 434 & 2.2 & 681 & 4.2 & 247 & 4.5 & 2705 \\
\hline-116.4 & 34 & 437 & 2.4 & 680 & 3 & 243 & 4 & 2701 \\
\hline-116.4 & 34.3 & 437 & 1.4 & 680 & 0.9 & 243 & 1.7 & 2637 \\
\hline-116.4 & 34.6 & 433 & 0.9 & 680 & 1.1 & 247 & 1.7 & 2549 \\
\hline-116.4 & 34.9 & 432 & 0.7 & 681 & 0.8 & 248 & 1.2 & 2435 \\
\hline-116.4 & 35.2 & 431 & 0.5 & 681 & 0.9 & 249 & 1 & 2180 \\
\hline-116.4 & 35.5 & 432 & 0.5 & 680 & 0.8 & 247 & 0.9 & 1895 \\
\hline-116.4 & 35.8 & 433 & 0.5 & 679 & 1 & 246 & 1.2 & 1724 \\
\hline-116.4 & 36.1 & 432 & 0.6 & 681 & 1.3 & 249 & 1.3 & 1338 \\
\hline-116.4 & 36.4 & 430 & 1 & 680 & 2.2 & 249 & 2.5 & 839 \\
\hline-116.4 & 36.7 & 430 & 1 & 676 & 1.6 & 245 & 1.9 & 549 \\
\hline-116.4 & 37 & 429 & 1 & 673 & 1.9 & 244 & 2.1 & 523 \\
\hline-116.4 & 37.3 & 428 & 1.1 & 673 & 4.1 & 245 & 4.1 & 552 \\
\hline-116.4 & 37.6 & 430 & 1.4 & 671 & 3.5 & 241 & 4 & 450 \\
\hline-116.4 & 37.9 & 435 & 2.3 & 689 & 2.5 & 254 & 2.6 & 252 \\
\hline-116.1 & 31 & 430 & 1.5 & 692 & 1.5 & 262 & 1.9 & 122 \\
\hline-116.1 & 31.3 & 433 & 1.1 & 687 & 1.5 & 254 & 1.7 & 378 \\
\hline-116.1 & 31.6 & 435 & 1.1 & 686 & 1.3 & 251 & 1.7 & 1084 \\
\hline-116.1 & 31.9 & 437 & 0.8 & 688 & 1 & 250 & 1.4 & 1483 \\
\hline-116.1 & 32.2 & 434 & 0.6 & 692 & 0.5 & 257 & 0.8 & 3066 \\
\hline-116.1 & 32.5 & 432 & 0.5 & 690 & 0.4 & 257 & 0.6 & 4359 \\
\hline-116.1 & 32.8 & 432 & 0.8 & 688 & 0.9 & 255 & 1.2 & 4314 \\
\hline-116.1 & 33.1 & 423 & 0.6 & 675 & 1.1 & 251 & 1.2 & 2952 \\
\hline-116.1 & 33.4 & 427 & 0.7 & 669 & 1.2 & 242 & 1.4 & 2812 \\
\hline-116.1 & 33.7 & 429 & 1.4 & 686 & 4.6 & 256 & 4.4 & 2845 \\
\hline-116.1 & 34 & 435 & 1.1 & 682 & 1.2 & 246 & 1.6 & 3138 \\
\hline-116.1 & 34.3 & 436 & 0.9 & 682 & 1.8 & 245 & 2 & 3095 \\
\hline-116.1 & 34.6 & 431 & 3.2 & 683 & 1.1 & 252 & 3.2 & 2794 \\
\hline-116.1 & 34.9 & 434 & 1 & 684 & 1 & 250 & 1.3 & 2147 \\
\hline-116.1 & 35.2 & 432 & 0.6 & 680 & 1.1 & 248 & 1.2 & 2101 \\
\hline-116.1 & 35.5 & 436 & 0.6 & 680 & 0.8 & 243 & 0.9 & 2044 \\
\hline
\end{tabular}




\begin{tabular}{|c|c|c|c|c|c|c|c|c|}
\hline-116.1 & 35.8 & 434 & 0.8 & 680 & 1.1 & 246 & 1.3 & 1700 \\
\hline-116.1 & 36.1 & 434 & 1 & 679 & 1 & 244 & 1.1 & 1178 \\
\hline-116.1 & 36.4 & 432 & 1 & 679 & 1.1 & 247 & 1.3 & 851 \\
\hline-116.1 & 36.7 & 433 & 2.1 & 679 & 1.4 & 245 & 2.5 & 561 \\
\hline-116.1 & 37 & 431 & 1.6 & 675 & 1.8 & 244 & 2.5 & 478 \\
\hline-116.1 & 37.3 & 431 & 3 & 676 & 2 & 245 & 3.7 & 521 \\
\hline-116.1 & 37.6 & 431 & 1.3 & 675 & 1.1 & 244 & 1.9 & 452 \\
\hline-116.1 & 37.9 & 435 & 2.2 & 679 & 2.3 & 244 & 3.5 & 232 \\
\hline-115.8 & 31 & 433 & 2.3 & 692 & 2.6 & 259 & 3 & 110 \\
\hline-115.8 & 31.3 & 430 & 1.3 & 681 & 1.9 & 251 & 2.3 & 495 \\
\hline-115.8 & 31.6 & 436 & 1.3 & 684 & 1 & 247 & 1.6 & 1213 \\
\hline-115.8 & 31.9 & 436 & 0.9 & 686 & 1.4 & 249 & 1.7 & 1934 \\
\hline-115.8 & 32.2 & 434 & 0.5 & 690 & 0.6 & 255 & 0.8 & 4730 \\
\hline-115.8 & 32.5 & 433 & 0.3 & 690 & 0.2 & 256 & 0.4 & 5798 \\
\hline-115.8 & 32.8 & 434 & 0.5 & 689 & 0.4 & 255 & 0.7 & 5624 \\
\hline-115.8 & 33.1 & 430 & 1.4 & 683 & 1.5 & 253 & 2.2 & 2971 \\
\hline-115.8 & 33.4 & 427 & 0.8 & 671 & 1.7 & 243 & 2 & 2337 \\
\hline-115.8 & 33.7 & 432 & 1.2 & 677 & 2.4 & 244 & 2.8 & 2700 \\
\hline-115.8 & 34 & 436 & 1.4 & 680 & 1.1 & 244 & 2 & 3014 \\
\hline-115.8 & 34.3 & 435 & 1.4 & 683 & 0.8 & 247 & 1.6 & 2933 \\
\hline-115.8 & 34.6 & 433 & 1.7 & 685 & 1 & 252 & 2 & 2522 \\
\hline-115.8 & 34.9 & 435 & 2.8 & 685 & 1.1 & 250 & 3.2 & 1974 \\
\hline-115.8 & 35.2 & 437 & 1.4 & 683 & 0.6 & 246 & 1.5 & 2106 \\
\hline-115.8 & 35.5 & 436 & 0.8 & 679 & 1 & 243 & 1.4 & 1637 \\
\hline-115.8 & 35.8 & 437 & 1.2 & 681 & 0.7 & 244 & 1.4 & 1474 \\
\hline-115.8 & 36.1 & 439 & 0.9 & 682 & 0.8 & 242 & 1.2 & 1127 \\
\hline-115.8 & 36.4 & 435 & 0.7 & 681 & 1 & 245 & 1.3 & 710 \\
\hline-115.8 & 36.7 & 438 & 4.2 & 680 & 1.6 & 242 & 4.3 & 451 \\
\hline-115.8 & 37 & 436 & 3 & 677 & 1.8 & 240 & 3.9 & 419 \\
\hline-115.8 & 37.3 & 433 & 1.4 & 679 & 1.6 & 246 & 2.1 & 395 \\
\hline-115.8 & 37.6 & 434 & 1.7 & 679 & 1.5 & 245 & 2.1 & 308 \\
\hline-115.8 & 37.9 & 434 & 2.9 & 677 & 1.8 & 243 & 3.3 & 151 \\
\hline-115.5 & 31 & 433 & 2.8 & 688 & 2.9 & 255 & 4.2 & 124 \\
\hline-115.5 & 31.3 & 430 & 1.1 & 677 & 1.1 & 247 & 1.6 & 593 \\
\hline-115.5 & 31.6 & 433 & 0.9 & 681 & 1.1 & 247 & 1.4 & 1351 \\
\hline-115.5 & 31.9 & 435 & 0.8 & 683 & 0.7 & 248 & 1.1 & 2006 \\
\hline-115.5 & 32.2 & 434 & 0.4 & 689 & 0.6 & 254 & 0.8 & 5460 \\
\hline-115.5 & 32.5 & 434 & 0.5 & 689 & 0.5 & 255 & 0.6 & 6868 \\
\hline-115.5 & 32.8 & 434 & 0.5 & 689 & 0.5 & 255 & 0.7 & 6217 \\
\hline-115.5 & 33.1 & 436 & 0.7 & 686 & 1.4 & 250 & 1.5 & 2800 \\
\hline-115.5 & 33.4 & 430 & 1.7 & 673 & 1 & 243 & 2.1 & 2050 \\
\hline-115.5 & 33.7 & 429 & 0.7 & 677 & 1.6 & 247 & 1.5 & 2558 \\
\hline-115.5 & 34 & 429 & 1.3 & 678 & 1.2 & 249 & 1.7 & 2801 \\
\hline-115.5 & 34.3 & 432 & 1.3 & 681 & 1 & 249 & 1.6 & 2557 \\
\hline-115.5 & 34.6 & 437 & 1.1 & 686 & 0.7 & 248 & 1.3 & 2172 \\
\hline-115.5 & 34.9 & 438 & 1.1 & 686 & 1.1 & 247 & 1.6 & 1780 \\
\hline-115.5 & 35.2 & 439 & 0.9 & 685 & 1 & 245 & 1.4 & 1698 \\
\hline-115.5 & 35.5 & 440 & 0.9 & 683 & 1 & 242 & 1.2 & 1258 \\
\hline-115.5 & 35.8 & 439 & 1.1 & 683 & 0.8 & 244 & 1.3 & 1161 \\
\hline-115.5 & 36.1 & 439 & 0.9 & 683 & 1.2 & 243 & 1.7 & 855 \\
\hline-115.5 & 36.4 & 439 & 1.1 & 680 & 1.6 & 241 & 1.8 & 470 \\
\hline-115.5 & 36.7 & 438 & 1.7 & 680 & 2.1 & 241 & 2.3 & 240 \\
\hline-115.5 & 37 & 436 & 4.2 & 683 & 1.5 & 246 & 4.4 & 212 \\
\hline-115.5 & 37.3 & 431 & 1.4 & 681 & 1.8 & 249 & 1.9 & 169 \\
\hline
\end{tabular}




\begin{tabular}{|c|c|c|c|c|c|c|c|c|}
\hline-115.5 & 37.6 & 432 & 1.5 & 681 & 2.2 & 249 & 2.5 & 152 \\
\hline-115.5 & 37.9 & 435 & 2.3 & 677 & 2.3 & 241 & 3.2 & 109 \\
\hline-115.2 & 31.3 & 428 & 1.3 & 676 & 1.1 & 248 & 2 & 612 \\
\hline-115.2 & 31.6 & 430 & 1.2 & 680 & 1.1 & 249 & 1.6 & 1299 \\
\hline-115.2 & 31.9 & 435 & 0.9 & 684 & 0.8 & 248 & 1 & 2087 \\
\hline-115.2 & 32.2 & 434 & 0.4 & 687 & 0.6 & 252 & 0.7 & 5301 \\
\hline-115.2 & 32.5 & 435 & 0.2 & 687 & 0.4 & 252 & 0.5 & 6804 \\
\hline-115.2 & 32.8 & 434 & 0.4 & 687 & 0.4 & 253 & 0.5 & 5727 \\
\hline-115.2 & 33.1 & 437 & 0.6 & 682 & 0.8 & 244 & 0.8 & 2354 \\
\hline-115.2 & 33.4 & 431 & 1.3 & 678 & 2 & 246 & 2.1 & 1936 \\
\hline-115.2 & 33.7 & 426 & 1.7 & 679 & 1.2 & 252 & 2 & 2072 \\
\hline-115.2 & 34 & 430 & 1.1 & 678 & 0.9 & 247 & 1.2 & 2132 \\
\hline-115.2 & 34.3 & 433 & 1.4 & 681 & 1.2 & 247 & 1.8 & 1796 \\
\hline-115.2 & 34.6 & 435 & 1.9 & 686 & 0.8 & 250 & 2 & 1627 \\
\hline-115.2 & 34.9 & 439 & 1 & 686 & 0.9 & 247 & 1.4 & 1389 \\
\hline-115.2 & 35.2 & 439 & 0.9 & 684 & 1.5 & 245 & 1.6 & 1101 \\
\hline-115.2 & 35.5 & 438 & 0.6 & 683 & 1 & 244 & 0.9 & 1051 \\
\hline-115.2 & 35.8 & 439 & 0.9 & 682 & 1.1 & 243 & 1.4 & 921 \\
\hline-115.2 & 36.1 & 439 & 1.2 & 684 & 1.5 & 244 & 2 & 492 \\
\hline-115.2 & 36.4 & 444 & 1.1 & 685 & 1.9 & 240 & 2.2 & 312 \\
\hline-115.2 & 36.7 & 441 & 2.6 & 681 & 3 & 240 & 3.8 & 144 \\
\hline-115.2 & 37.3 & 430 & 0.9 & 682 & 1.3 & 251 & 1.6 & 115 \\
\hline-114.9 & 31.3 & 425 & 1.2 & 676 & 2 & 251 & 2.2 & 524 \\
\hline-114.9 & 31.6 & 431 & 0.9 & 680 & 1.7 & 249 & 1.9 & 1102 \\
\hline-114.9 & 31.9 & 437 & 0.9 & 681 & 0.9 & 244 & 1.4 & 1981 \\
\hline-114.9 & 32.2 & 436 & 0.5 & 685 & 0.5 & 248 & 0.7 & 3995 \\
\hline-114.9 & 32.5 & 436 & 0.4 & 685 & 0.3 & 248 & 0.6 & 4783 \\
\hline-114.9 & 32.8 & 436 & 0.5 & 684 & 0.4 & 248 & 0.7 & 4269 \\
\hline-114.9 & 33.1 & 437 & 0.5 & 680 & 0.6 & 243 & 0.9 & 1837 \\
\hline-114.9 & 33.4 & 433 & 0.9 & 682 & 1.5 & 248 & 1.8 & 1666 \\
\hline-114.9 & 33.7 & 429 & 1.4 & 683 & 0.7 & 253 & 1.4 & 1665 \\
\hline-114.9 & 34 & 427 & 1.3 & 680 & 1.9 & 252 & 2.1 & 1557 \\
\hline-114.9 & 34.3 & 430 & 0.9 & 680 & 1.8 & 250 & 2 & 1112 \\
\hline-114.9 & 34.6 & 431 & 0.9 & 686 & 1.5 & 254 & 1.9 & 936 \\
\hline-114.9 & 34.9 & 434 & 1.4 & 686 & 0.5 & 251 & 1.6 & 854 \\
\hline-114.9 & 35.2 & 435 & 1.4 & 686 & 1 & 251 & 1.5 & 772 \\
\hline-114.9 & 35.5 & 435 & 0.8 & 684 & 1 & 248 & 1.4 & 725 \\
\hline-114.9 & 35.8 & 436 & 0.9 & 685 & 1.5 & 249 & 1.9 & 493 \\
\hline-114.9 & 36.1 & 438 & 0.8 & 684 & 2 & 245 & 2.1 & 337 \\
\hline-114.9 & 36.4 & 443 & 3 & 683 & 3.5 & 239 & 4.5 & 166 \\
\hline-114.6 & 31.3 & 423 & 1.6 & 0 & 0 & 0 & 0 & 433 \\
\hline-114.6 & 31.6 & 429 & 1.4 & 681 & 1.7 & 252 & 2.1 & 811 \\
\hline-114.6 & 31.9 & 436 & 1 & 679 & 1 & 243 & 1.5 & 1467 \\
\hline-114.6 & 32.2 & 437 & 0.6 & 684 & 0.5 & 247 & 0.6 & 2764 \\
\hline-114.6 & 32.5 & 436 & 0.5 & 683 & 0.8 & 246 & 0.7 & 3216 \\
\hline-114.6 & 32.8 & 437 & 0.5 & 682 & 0.7 & 245 & 0.9 & 2757 \\
\hline-114.6 & 33.1 & 435 & 0.8 & 681 & 1.2 & 245 & 1.2 & 1379 \\
\hline-114.6 & 33.4 & 435 & 0.8 & 684 & 1.4 & 249 & 1.8 & 1368 \\
\hline-114.6 & 33.7 & 432 & 1 & 683 & 1 & 251 & 1.2 & 1224 \\
\hline-114.6 & 34 & 424 & 2.6 & 684 & 0.9 & 259 & 2.7 & 1034 \\
\hline-114.6 & 34.3 & 428 & 1.2 & 682 & 1.4 & 254 & 2 & 711 \\
\hline-114.6 & 34.6 & 431 & 0.9 & 685 & 0.9 & 254 & 1.2 & 552 \\
\hline-114.6 & 34.9 & 433 & 1.6 & 687 & 0.6 & 254 & 1.9 & 597 \\
\hline-114.6 & 35.2 & 431 & 1.4 & 685 & 0.6 & 253 & 1.6 & 609 \\
\hline
\end{tabular}




\begin{tabular}{|c|c|c|c|c|c|c|c|c|}
\hline-114.6 & 35.5 & 432 & 1.6 & 682 & 0.9 & 250 & 1.9 & 428 \\
\hline-114.6 & 35.8 & 434 & 1.1 & 684 & 0.8 & 249 & 1.3 & 366 \\
\hline-114.6 & 36.1 & 435 & 1.1 & 682 & 1.2 & 246 & 1.6 & 236 \\
\hline-114.6 & 36.4 & 431 & 2.8 & 680 & 3.5 & 249 & 4.3 & 105 \\
\hline-114.3 & 31.3 & 414 & 3.1 & 684 & 3.1 & 270 & 4.2 & 245 \\
\hline-114.3 & 31.6 & 429 & 1.1 & 679 & 1.2 & 250 & 1.6 & 562 \\
\hline-114.3 & 31.9 & 432 & 2 & 680 & 1.1 & 247 & 2.5 & 905 \\
\hline-114.3 & 32.2 & 439 & 0.8 & 677 & 1 & 238 & 1.5 & 1504 \\
\hline-114.3 & 32.5 & 437 & 0.4 & 678 & 0.8 & 240 & 0.9 & 1766 \\
\hline-114.3 & 32.8 & 437 & 0.9 & 679 & 1 & 242 & 1.3 & 1307 \\
\hline-114.3 & 33.1 & 431 & 1.1 & 676 & 1.2 & 245 & 1.6 & 876 \\
\hline-114.3 & 33.4 & 430 & 1.1 & 683 & 1.4 & 252 & 1.9 & 1063 \\
\hline-114.3 & 33.7 & 429 & 1.4 & 684 & 1.1 & 254 & 1.7 & 873 \\
\hline-114.3 & 34 & 431 & 1.2 & 687 & 1 & 256 & 1.7 & 677 \\
\hline-114.3 & 34.3 & 429 & 1 & 686 & 0.9 & 256 & 1.4 & 376 \\
\hline-114.3 & 34.6 & 431 & 1.2 & 687 & 1.1 & 256 & 1.6 & 446 \\
\hline-114.3 & 34.9 & 432 & 3.5 & 687 & 1.3 & 254 & 3.8 & 402 \\
\hline-114.3 & 35.2 & 432 & 3.6 & 683 & 1.1 & 251 & 4.1 & 412 \\
\hline-114.3 & 35.5 & 431 & 2.3 & 684 & 0.9 & 252 & 2.5 & 409 \\
\hline-114.3 & 35.8 & 432 & 1.1 & 681 & 1 & 248 & 1.3 & 333 \\
\hline-114.3 & 36.1 & 435 & 2.6 & 680 & 1.8 & 245 & 3.1 & 162 \\
\hline-114 & 31.3 & 0 & 0 & 687 & 1.2 & 0 & 0 & 174 \\
\hline-114 & 31.6 & 432 & 2.9 & 682 & 2.7 & 250 & 3.8 & 497 \\
\hline-114 & 31.9 & 426 & 3.1 & 681 & 2.2 & 255 & 4 & 645 \\
\hline-114 & 32.2 & 427 & 2.3 & 680 & 1.8 & 253 & 3 & 656 \\
\hline-114 & 32.5 & 425 & 1.1 & 678 & 1 & 253 & 1.6 & 614 \\
\hline-114 & 32.8 & 426 & 1.2 & 677 & 1 & 251 & 1.7 & 643 \\
\hline-114 & 33.1 & 425 & 1.4 & 674 & 0.9 & 249 & 1.6 & 701 \\
\hline-114 & 33.4 & 428 & 1.3 & 676 & 1.4 & 248 & 1.7 & 772 \\
\hline-114 & 33.7 & 426 & 0.9 & 682 & 1.6 & 255 & 1.9 & 696 \\
\hline-114 & 34 & 428 & 0.9 & 683 & 1.3 & 255 & 1.8 & 569 \\
\hline-114 & 34.3 & 433 & 1.3 & 688 & 1.4 & 254 & 1.8 & 337 \\
\hline-114 & 34.6 & 434 & 1.3 & 689 & 1.1 & 255 & 1.7 & 371 \\
\hline-114 & 34.9 & 438 & 2.1 & 684 & 1.3 & 246 & 2.6 & 373 \\
\hline-114 & 35.2 & 432 & 3.8 & 682 & 1.2 & 249 & 4.1 & 434 \\
\hline-114 & 35.5 & 431 & 2.3 & 682 & 2.3 & 250 & 3.1 & 377 \\
\hline-114 & 35.8 & 432 & 1.5 & 679 & 2.3 & 247 & 2.5 & 259 \\
\hline-113.7 & 31.6 & 436 & 1.5 & 682 & 3.9 & 245 & 4.1 & 359 \\
\hline-113.7 & 31.9 & 431 & 2 & 681 & 1.9 & 249 & 2.6 & 632 \\
\hline-113.7 & 32.2 & 428 & 3.3 & 677 & 1.3 & 249 & 3.5 & 651 \\
\hline-113.7 & 32.5 & 425 & 1.9 & 679 & 1.1 & 254 & 2.2 & 506 \\
\hline-113.7 & 32.8 & 425 & 1.7 & 678 & 0.8 & 253 & 1.9 & 492 \\
\hline-113.7 & 33.1 & 424 & 2.2 & 677 & 1 & 253 & 2.1 & 565 \\
\hline-113.7 & 33.4 & 426 & 1.2 & 676 & 0.9 & 250 & 1.6 & 764 \\
\hline-113.7 & 33.7 & 427 & 1 & 675 & 1.2 & 247 & 1.5 & 604 \\
\hline-113.7 & 34 & 425 & 1 & 678 & 2.9 & 252 & 3.2 & 482 \\
\hline-113.7 & 34.3 & 428 & 1.3 & 686 & 1.6 & 257 & 1.8 & 333 \\
\hline-113.7 & 34.6 & 437 & 2.6 & 685 & 1.7 & 248 & 3 & 307 \\
\hline-113.7 & 34.9 & 435 & 2.6 & 684 & 3 & 249 & 4.1 & 353 \\
\hline-113.7 & 35.2 & 431 & 4.1 & 679 & 1.5 & 248 & 4.2 & 341 \\
\hline-113.7 & 35.5 & 428 & 2.2 & 679 & 1.9 & 251 & 3.4 & 295 \\
\hline-113.7 & 35.8 & 429 & 1.9 & 678 & 3.7 & 248 & 4.1 & 197 \\
\hline-113.4 & 31.6 & 435 & 1.5 & 693 & 3.3 & 257 & 3.9 & 293 \\
\hline-113.4 & 31.9 & 432 & 1.4 & 682 & 2.6 & 250 & 2.9 & 484 \\
\hline
\end{tabular}




\begin{tabular}{|c|c|c|c|c|c|c|c|c|}
-113.4 & 32.2 & 431 & 2.3 & 678 & 1.3 & 247 & 2.8 & 559 \\
\hline-113.4 & 32.5 & 429 & 2 & 678 & 1.5 & 248 & 2.3 & 397 \\
\hline-113.4 & 32.8 & 430 & 1.5 & 677 & 1.2 & 247 & 2.1 & 439 \\
\hline-113.4 & 33.1 & 425 & 2 & 677 & 1.2 & 252 & 2.3 & 463 \\
\hline-113.4 & 33.4 & 422 & 3.1 & 675 & 1.1 & 252 & 3.1 & 672 \\
\hline-113.4 & 33.7 & 426 & 1.2 & 674 & 0.9 & 247 & 1.5 & 676 \\
\hline-113.4 & 34 & 426 & 0.8 & 672 & 2 & 245 & 2.3 & 416 \\
\hline-113.4 & 34.3 & 429 & 2.1 & 683 & 3.4 & 254 & 4.1 & 232 \\
\hline-113.4 & 34.6 & 442 & 4.1 & 685 & 2.3 & 243 & 4 & 248 \\
\hline-113.4 & 34.9 & 433 & 2.6 & 681 & 1.9 & 247 & 3.1 & 289 \\
\hline-113.4 & 35.2 & 432 & 1.6 & 676 & 2.1 & 244 & 2.6 & 284 \\
\hline-113.4 & 35.5 & 430 & 2.4 & 674 & 2.6 & 244 & 3.4 & 236 \\
\hline-113.4 & 35.8 & 430 & 2.6 & 689 & 3.1 & 258 & 4.2 & 148 \\
\hline-113.1 & 31.6 & 432 & 1.4 & 698 & 3.3 & 265 & 3.7 & 231 \\
\hline-113.1 & 31.9 & 432 & 1.5 & 679 & 2.6 & 247 & 3.2 & 286 \\
\hline-113.1 & 32.2 & 433 & 1.2 & 678 & 2.8 & 245 & 3.1 & 338 \\
\hline-113.1 & 32.5 & 433 & 2.5 & 677 & 2.8 & 244 & 3.8 & 240 \\
\hline-113.1 & 32.8 & 426 & 3.8 & 679 & 1.6 & 252 & 4.2 & 342 \\
\hline-113.1 & 33.1 & 431 & 2.9 & 673 & 1.5 & 241 & 3.2 & 414 \\
\hline-113.1 & 33.4 & 426 & 3.6 & 675 & 1.4 & 249 & 3.5 & 501 \\
\hline-113.1 & 33.7 & 426 & 1.9 & 673 & 2 & 247 & 2.1 & 460 \\
\hline-113.1 & 34 & 424 & 1.5 & 671 & 2.2 & 247 & 2.2 & 338 \\
\hline-113.1 & 34.3 & 426 & 3.2 & 683 & 2.7 & 257 & 3.6 & 161 \\
\hline-113.1 & 34.6 & 0 & 0 & 684 & 2.1 & 0 & 0 & 168 \\
\hline-113.1 & 34.9 & 0 & 0 & 679 & 1.7 & 0 & 0 & 192 \\
\hline-113.1 & 35.2 & 427 & 1.6 & 676 & 2.2 & 249 & 2.8 & 193 \\
\hline-113.1 & 35.5 & 427 & 1.5 & 680 & 3.7 & 252 & 3.8 & 168 \\
\hline
\end{tabular}

156 